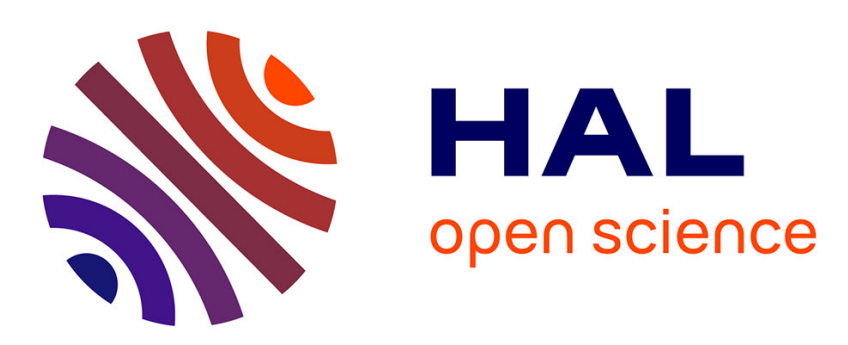

\title{
Wavenumber explicit convergence analysis for finite element discretizations of general wave propagation problems
}

Théophile Chaumont-Frelet, Serge Nicaise

\section{- To cite this version:}

Théophile Chaumont-Frelet, Serge Nicaise. Wavenumber explicit convergence analysis for finite element discretizations of general wave propagation problems. IMA Journal of Numerical Analysis, 2019, 10.1093/imanum/drz020. hal-01685388v2

\section{HAL Id: hal-01685388 \\ https://hal.inria.fr/hal-01685388v2}

Submitted on 11 Dec 2018

HAL is a multi-disciplinary open access archive for the deposit and dissemination of scientific research documents, whether they are published or not. The documents may come from teaching and research institutions in France or abroad, or from public or private research centers.
L'archive ouverte pluridisciplinaire HAL, est destinée au dépôt et à la diffusion de documents scientifiques de niveau recherche, publiés ou non, émanant des établissements d'enseignement et de recherche français ou étrangers, des laboratoires publics ou privés. 


\title{
WAVENUMBER EXPLICIT CONVERGENCE ANALYSIS FOR FINITE ELEMENT DISCRETIZATIONS OF GENERAL WAVE PROPAGATION PROBLEMS
}

\author{
T. CHAUMONT-FRELET ${ }^{1}$ AND S. NICAISE ${ }^{2}$
}

\begin{abstract}
We analyze the convergence of finite element discretizations of time-harmonic wave propagation problems. We propose a general methodology to derive stability conditions and error estimates that are explicit with respect to the wavenumber $k$. This methodology is formally based on an expansion of the solution in powers of $k$, which permits to split the solution into a regular, but oscillating part, and another component that is rough, but behaves nicely when the wavenumber increases. The method is developed in its full generality and is illustrated by three particular cases: the elastodynamic system, the convected Helmholtz equation, and the acoustic Helmholtz equation in homogeneous and heterogeneous media. Numerical experiments are provided which confirm that the stability conditions and error estimates are sharp.
\end{abstract}

\section{INTRODUCTION}

The propagation of waves is used in a wide range of applications including resource prospection, radar, and medical imaging (see, e.g., Chavent et al., 2012; Colton \& Kress, 2012). These applications motivate the development of efficient discretization techniques to approximate the solutions to wave propagation problems in an accurate and robust fashion.

In this work, we focus on time-harmonic wave propagation problems. A number of discretization techniques are available to approximate their solutions, but for the sake of simplicity, we only consider finite element methods here. Nevertheless, a great part of the material we present shall be useful in the understanding of other approximation strategies, such as finite difference or integral equation approaches.

The so-called "pollution effect" is a key concept to understand the performance and limitations of finite element discretizations (Ihlenburg \& Babuška, 1995, 1997). When the number of wavelength inside the propagation domain is important, the numerical solution is only meaningful under restrictive conditions on the mesh size. This effect is manifested by a gap between the error of the best approximation the finite element scheme is capable of (e.g. the interpolant) and the error of the numerical solution that is actually produced. This gap becomes more important as the frequency increases, unless additional discretization points per wavelength are employed. As a result, finite element methods require a large number of degrees of freedom to produce accurate solutions to high-frequency problems, in which the number of wavelength inside the domain is important.

As the pollution effect is characterized by a gap between the best approximation error, and the finite element one, it is related to a lack of stability of the finite element scheme. This lack of stability is typical of wave problems, since the sesquilinear forms that naturally appear by integration by parts are not coercive. Because of this lack of coercivity, the quasi-optimality of the finite element solution in the energy norm is not guaranteed for arbitrary meshes. Actually, it is shown in the literature that quasi-optimality of the scheme is achieved only in an "asymptotic range" (i.e. for small enough mesh sizes) that depends on the frequency and the discretization order.

The behaviour of the asymptotic range with respect to the frequency, the mesh size, and the discretization order is the key to understand the efficiency of a finite element method. For the

\footnotetext{
${ }^{1}$ BCAM - Basque Center for Applied Mathematics - Alameda Mazarredo, 14, 48009 Bilbao, Bizkaia

2 Univ. Valenciennes, EA 4015 - LAMAV - Laboratoire de Mathématiques et leurs Applications de Valenciennes, FR CNRS 2956, F-59313 Valenciennes, France
} 
acoustic Helmholtz equation in smooth domains, the asymptotic range for $h p$-finite element methods has been characterized in a sequence of papers by J.M. Melenk and collaborators (Esterhazy \& Melenk, 2012; Melenk \& Sauter, 2010, 2011). The main result states that if

$$
\frac{k h}{p} \leq C_{1}, \quad k\left(\frac{k h}{p}\right)^{p} \leq C_{2},
$$

then the finite element solution is quasi-optimal. In (1), $k$ is the wavenumber, $h$ is the mesh size, $p$ is the discretization order and $C_{1}$ and $C_{2}$ are two constants independent of these parameters.

When considering a fixed $p$, we deduce from (1) that the condition $k^{p+1} h^{p} \leq C$ must be fulfilled to preserve quasi-optimality. In other words, the asymptotic range in which the finite element solution is quasi-optimal is characterized by

$$
h \leq h^{\star}(k)=C k^{-1-1 / p} .
$$

We also see that the number of element per wavelength $(k h)^{-1}$ must be increased for increasing frequencies since (1) implies that

$$
(k h)^{-1} \geq C k^{1 / p} .
$$

In addition, since the constant $C_{1}$ and $C_{2}$ are independent of $p$, stability conditions for varying $p$ are also possible. Namely, stability is ensured if

$$
\frac{k h}{p}=C_{3}, \quad p \geq C_{4} \log k,
$$

which means that the number of discretization points per wavelength $p /(k h)$ is kept constant while the discretization order increases logarithmically with the wavenumber.

The main ingredient to obtain (1) is a splitting of the solution to the Helmholtz equation into a regular but oscillating part, and a rough component that behaves "nicely" for large frequencies. This splitting is based on a filtering of the datum in the Fourier domain, and treating the low and high frequency contents with distinct tools.

While this analysis is sharp and valid for arbitrary order $p$, it is currently limited to homogeneous media. In particular, results are available for the acoustic Helmholtz equation posed in either

- a bounded analytic domain with impedance boundary conditions (Melenk \& Sauter, 2011, Case I), or

- a 2D polygonal domain with impedance boundary conditions (Melenk \& Sauter, 2011, Case II) and (Esterhazy \& Melenk, 2012, Equation 9), or

- the exterior of an analytic domain with Dirichlet boundary conditions on the scatterer and the Sommerfeld radiation condition at infinity (Melenk \& Sauter, 2011, Case III).

We also mention similar results for the Maxwell system set in a ball with transparent boundary conditions, see Melenk \& Sauter (2018).

Our main contribution is to propose another splitting approach to provide (1). Such a splitting is defined using a sequence of solutions to elliptic boundary value problems. This sequence is defined recursively, and the iterates exhibit increasing regularity. The main advantage of this splitting is that it requires less restrictive assumptions on the domain and the wave operator. Indeed, the only important assumption we make is that the elliptic part of the wave operator satisfy a regularity shift (see Definition 1). As a result, as shown in Section 2, our result applies to a variety of wave propagation problems. We illustrate this in Sections 3, 4, 5 and 6 by applying our result to the particular cases of the elastodynamic system, the convected Helmholtz equation and the acoustic Helmholtz equation in homogeneous or heterogeneous media.

While our analysis applies to a wider range of problems, it is currently limited in the sense that the constants $C_{1}$ and $C_{2}$ we obtain in (1) depends on $p$. As a result, we recover the same result for a fixed $p$ : in particular (2) and (3). However, our analysis does not cover varying $p$, and thus, a result like (4) is out of the scope of the present article.

Our work is organized as follows. The splitting of the solution and the derivation of stability result (1) are presented for a general wave operator in Section 2. This analysis is illustrated in Sections 3, 4, 5 and 6 by considering the elastodynamic system, the convected Helmholtz 
equation and the acoustic Helmholtz equation in homogeneous or heterogeneous media. In all cases, except for the acoustic Helmholtz equation in homogeneous media, numerical experiments are presented, that confirm that the theoretical results are sharp. In the conclusion, we state the main consequences of our results as well as possible extensions.

We finish this section with some notations used in the remainder of the paper. The set of non negative integers will be denoted by $\mathbb{N}$, i. e., $\mathbb{N}=\{0,1,2, \cdots\}$. For a bounded domain $D$, the usual norm and semi-norm of $H^{s}(D)(s \geq 0)$ are denoted by $\|\cdot\|_{s, D}$ and $|\cdot|_{s, D}$, respectively. Furthermore, the notation $A \lesssim B$ (resp. $A \gtrsim B$ ) means the existence of a positive constant $C_{1}$ (resp. $C_{2}$ ), which is independent of $k, h, A$ and $B$ such that $A \leq C_{1} B$ (resp. $A \geq C_{2} B$ ). The notation $A \sim B$ means that $A \lesssim B$ and $A \gtrsim B$ hold simultaneously.

\section{General Settings}

2.1. Splitting of the solution. In the following, we consider a bounded domain $\Omega \subset \mathbb{R}^{N}(N=2$ or 3) and a general linear elliptic system $\mathcal{L}_{2}$ of order 2 and size $S \geq 1$ that acts on either scalar (if $S=1$ ) or vectorial functions (if $S \geq 2$ ) of $\Omega$. Since we want to allow operators with piecewise smooth coefficients, we suppose given a finite "partition" $\Omega_{q}, q \in \mathcal{Q}$ of $\Omega$ that satisfies the following properties: $\mathcal{Q}$ is a finite set, each $\Omega_{q}$ is a bounded open domain with a $C^{\gamma+1,1}$ boundary, with $\gamma \in \mathbb{N}$, except eventually at a finite number of points called corners of $\Omega_{q}$, they are pairwise disjoint and

$$
\bar{\Omega}=\cup_{q \in \mathcal{Q}} \overline{\Omega_{q}} .
$$

The set of corners of all $\Omega_{q}$ forms the set of corners of $\Omega$ and is denoted by $\mathcal{C}$. The case $\mathcal{Q}$ equals to a singleton and $\mathcal{C}=\emptyset$ corresponds to the situation of a single domain with a $C^{\gamma+1,1}$ boundary and is not excluded. Similarly, the case of several subdomains $\Omega_{q}$ with $C^{\gamma+1,1}$ boundaries (hence $\mathcal{C}=\emptyset)$ is also covered. We further suppose fixed two open disjoint parts $\Gamma_{A}$ and $\Gamma_{D}$ of the boundary $\partial \Omega$ of $\Omega$ such that

$$
\partial \Omega=\bar{\Gamma}_{A} \cup \bar{\Gamma}_{D}
$$

We also suppose fixed two boundary operators $\mathcal{B}_{D}$ and $\mathcal{B}_{A}$ defined respectively on $\Gamma_{D}$ and $\Gamma_{A}$. $\mathcal{B}_{D}$ is supposed to be the natural Dirichlet operator so that it can be identified with the trace operator (with constant coefficients), while $\mathcal{B}_{A}$ is supposed to be an operator of order 1 (typically the Neumann boundary operator associated with $\mathcal{L}_{2}$ plus possibly a zero order term).

We now introduce appropriate Sobolev spaces related to the partition of $\Omega$, namely for all $l \in \mathbb{N}$, we set

$$
\begin{array}{r}
P H^{l}(\Omega)=\left\{f \in L^{2}(\Omega): f_{q}:=f_{\mid \Omega_{q}} \in H^{l}\left(\Omega_{q}\right), \forall q \in \mathcal{Q}\right\}, \\
P H^{l+1 / 2}\left(\Gamma_{A}\right)=\left\{g \in L^{2}\left(\Gamma_{A}\right): g_{\mid \Gamma_{A} \cap \partial \Omega_{q}} \in H^{l+1 / 2}\left(\Gamma_{A} \cap \partial \Omega_{q}\right), \forall q \in \mathcal{Q}\right\},
\end{array}
$$

that are Hilbert spaces for their natural norms $\|\cdot\|_{\mathcal{Q}, l+2, \Omega}$ and $\|\cdot\|_{\mathcal{Q}, l+1 / 2, \Gamma_{A}}$.

We are first concerned with regularity (shift) results for the boundary value problem

$$
\begin{cases}\mathcal{L}_{2} \tilde{u}=\tilde{f} & \text { in } \Omega, \\ \mathcal{B}_{A} \tilde{u}=\tilde{g} & \text { on } \Gamma_{A}, \\ \mathcal{B}_{D} \tilde{u}=0 & \text { on } \Gamma_{D},\end{cases}
$$

with $\tilde{f} \in P H^{l}(\Omega)$ and $\tilde{g} \in P H^{l+1 / 2}\left(\Gamma_{A}\right)$. Note that, in case of discontinuous coefficients, the differential equation $\mathcal{L}_{2} \tilde{u}=\tilde{f}$ has to be interpretated in the sense that it holds on each subdomain $\Omega_{p}$ added with some transmission conditions that are the "continuity" (in the sense of trace) of $\tilde{u}$ across the interface $\overline{\Omega_{q}} \cap \overline{\Omega_{q^{\prime}}}$ and the continuity of the flux (in the sense of trace) across the interface, see for instance Nicaise \& Sändig (1994a).

When each subdomain $\Omega_{q}$ has a smooth boundary of class $C^{\gamma+1,1}$ (thus $\mathcal{C}=\emptyset$ ), we can expect that the solution $\tilde{u}$ to $(5)$ belongs to $P H^{l+2}(\Omega)$ if $\tilde{f} \in P H^{l}(\Omega)$ and $\tilde{g} \in P H^{l+1 / 2}\left(\Gamma_{A}\right)$ for all $l \leq \gamma$. Here, we consider the more complicated situation where the boundary of each $\Omega_{q}$ is allowed to exhibit corners. In the two dimensional case, we can consider general polygonal partitions for instance. In the three dimensional case, we allow corners, but for simplicity, edges are excluded.

If the partition $\mathcal{Q}$ exhibits corners, a framework that is more general than the broken Sobolev spaces $P H^{l}(\Omega)$ is required. According to Kondratiev's theory, Kondrat'ev (1967) (see also Dauge 
(1988); Grisvard (1985); Nicaise (1993); Nicaise \& Sändig (1994a)), the solution of (5) may exhibit singularities near these corners. For the sake of simplicity we assume that each $\Omega_{p}$ is straight near each corner $c$, in the sense that $\Omega_{p}$ coincides with an infinite cone

$$
K_{q, c}=\left\{r_{c} \vartheta_{c} ; r_{c}>0 \text { and } \vartheta_{c} \in G_{q, c}\right\},
$$

where $\left(r_{c}, \vartheta_{c}\right)$ are the spherical coordinates centred at $c$ and $G_{q, c}$ is a subset of the unit sphere with a $C^{1,1}$ boundary. We then set

$$
G_{c}=\bigcup_{q \in \mathcal{Q}, c \in \overline{\Omega_{q}}} G_{q, c}, \quad K_{c}=\bigcup_{q \in \mathcal{Q}, c \in \overline{\Omega_{q}}} K_{q, c}
$$

and, as before, denote by $P H^{2}\left(G_{c}\right)$ the space of functions in $L^{2}\left(G_{c}\right)$ that are in $H^{2}$ on each subdomain $G_{p, c}$.

Now for each corner $c$, we introduce the space $S_{c}^{\lambda}(\Omega)$ of quasi-homogeneous functions of exponent $\lambda \in \mathbb{C}$ defined as follows

$$
S_{c}^{\lambda}:=\left\{u \in L^{2}(\Omega) ; u=\eta_{c} r_{c}^{\lambda} \sum_{j=0}^{q}(\log r)^{j} \varphi_{j}\left(\vartheta_{c}\right), \varphi_{j} \in H^{1}\left(G_{c}\right)^{S} \cap P H^{2}\left(G_{c}\right)^{S}, q \in \mathbb{N}\right\},
$$

where $\eta_{c}$ is a cut-off function equal to 1 in a ball $B(c, \varepsilon)$ and equal to 0 outside $B(c, 2 \varepsilon)$ with $\varepsilon>0$ small enough (so that it is zero near the other corners of $\Omega$ ). We furthermore introduce the set of singular exponents $\Lambda_{c}$ as the set of complex numbers $\lambda$ such that there exists a solution $\varphi \in H^{1}\left(G_{c}\right)^{S} \cap P H^{2}\left(G_{c}\right)^{S}$ of

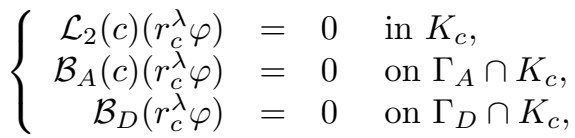

where $\mathcal{L}_{2}(c)$ (resp. $\left.\mathcal{B}_{A}(c)\right)$ is the principal part of $\mathcal{L}_{2}$ frozen (piecewisely) at $c$. According to the previous references, $\Lambda_{c}$ is a discrete set made of isolated points. Let us now introduce the set of singular functions associated with (5). For all $j \in \mathbb{N}$, each corner $c$, and any $\lambda \in \Lambda_{c}$ such that $\operatorname{Re} \lambda \in\left(1-\frac{N}{2}, j+2-\frac{N}{2}\right)$, we define $j(\lambda)$ as the largest integer such that $j(\lambda)<j+2-\frac{N}{2}-\operatorname{Re} \lambda$, we denote by

$$
\begin{aligned}
\mathcal{S}_{c, j}^{\lambda}=\left\{S^{\lambda} \in \operatorname{span}_{\ell=0}^{j(\lambda)} S_{c}^{\lambda+\ell} \backslash P H^{j+2}(\Omega) ;\right. & \text { satisfying }(7) \text { below }\} \\
& \begin{cases}\mathcal{L}_{2} S^{\lambda} \in P H^{j}(\Omega), \\
\mathcal{B}_{A} S^{\lambda} \in P H^{j+\frac{1}{2}}\left(\Gamma_{A}\right), \\
\mathcal{B}_{D} S^{\lambda}=0 & \text { on } \Gamma_{D} .\end{cases}
\end{aligned}
$$

We finally denote by $\left\{S_{c, j, q}^{\lambda}\right\}_{q=1}^{N_{c, j}^{\lambda}}$ a basis of $\mathcal{S}_{c, j}^{\lambda}$.

Definition 1. We will say that the triplet $\left(\mathcal{L}_{2}, \mathcal{B}_{D}, \mathcal{B}_{A}\right)$ satisfies the shift property at the order $\gamma+2$ for a natural number $\gamma$ when, for any natural number $l$ such that $0 \leq l \leq \gamma$, if $(\tilde{f}, \tilde{g}) \in$ $P H^{l}(\Omega)^{S} \times P H^{l+1 / 2}\left(\Gamma_{A}\right)^{S}$, then there exists a unique solution $\tilde{u} \in H^{1}(\Omega)^{S}$ of (5) such that $\tilde{u}$ admits the splitting

$$
\tilde{u}=\tilde{u}_{\mathrm{reg}}+\sum_{c \in \mathcal{C}} \sum_{\lambda \in \Lambda_{c}: \operatorname{Re}} \sum_{\lambda \in\left(1-\frac{N}{2}, l+2-\frac{N}{2}\right)} \sum_{q=1}^{N_{c, l}^{\lambda}} c_{c, q}^{\lambda} S_{c, l, q}^{\lambda},
$$

with $\tilde{u}_{\text {reg }} \in P H^{l+2}(\Omega)^{S}$ and $c_{c, q}^{\lambda} \in \mathbb{C}$ such that

$$
\|\tilde{u}\|_{\mathcal{Q}, l+2, \Omega}+\sum_{c \in \mathcal{C}} \sum_{\lambda \in \Lambda_{c}: \operatorname{Re}} \sum_{\lambda \in\left(1-\frac{N}{2}, l+2-\frac{N}{2}\right)}^{N_{c, l}^{\lambda}}\left|c_{q=1}^{\lambda}\right| \lesssim\|\tilde{f}\|_{\mathcal{Q}, l, \Omega}+\|\tilde{g}\|_{\mathcal{Q}, l+1 / 2, \Gamma_{A}} .
$$


Remark 1. If we consider subdomains with smooth boundaries of class $C^{\gamma+1,1}$, we can simply replace the statement of Definition 1 by $\tilde{u} \in P H^{l+2}(\Omega)^{S}$ and

$$
\|\tilde{u}\|_{\mathcal{Q}, l+2, \Omega} \lesssim\|\tilde{f}\|_{\mathcal{Q}, l, \Omega}+\|\tilde{g}\|_{\mathcal{Q}, l+1 / 2, \Gamma_{A}} .
$$

We now consider a time-harmonic wave propagation problem associated with the operator $\mathcal{L}_{2}$. We assume a complex wave number $\kappa=k-i \delta$ where $\delta$ is a fixed read number, and $k \geq 1$ can be arbitrary large. We look for $u \in H^{1}(\Omega)^{S}$ solution to

$$
\left\{\begin{aligned}
-\kappa^{2} \mathcal{L}_{0} u-i \kappa \mathcal{L}_{1} u+\mathcal{L}_{2} u & =f & & \text { in } \Omega, \\
\mathcal{B}_{A} u & =i \kappa A u & & \text { on } \Gamma_{A}, \\
\mathcal{B}_{D} u & =0 & & \text { on } \Gamma_{D},
\end{aligned}\right.
$$

where $f \in L^{2}(\Omega)^{S}$ is a given source term and $A$ is a matrix-valued function (or simply a scalar function) with the regularity $A \in P C^{\gamma, 1}\left(\Gamma_{A}, \mathcal{M}\left(\mathbb{R}^{S}\right)\right)\left(P C\right.$ meaning that its restriction to $\Gamma_{A} \cap \partial \Omega_{q}$ is in $C^{\gamma, 1}$ for each $q \in \mathcal{Q}$ ) and that is independent of $k$. In addition, $\mathcal{L}_{0}$ and $\mathcal{L}_{1}$ are linear operators satisfying

$$
\left\|\mathcal{L}_{0} v\right\|_{\mathcal{Q}, l, \Omega} \lesssim\|v\|_{\mathcal{Q}, l, \Omega}, \quad\left\|\mathcal{L}_{1} v\right\|_{\mathcal{Q}, l-1, \Omega} \lesssim\|v\|_{\mathcal{Q}, l, \Omega}
$$

for $l \in\{0, \ldots, \gamma+1\}$ and $v \in P H^{l}(\Omega)^{S}$.

We point out that all operators involved in the definition of system (9) are independent of $\kappa$. It means that system (9) only depends on the wavenumber through explicit mentions of $\kappa$.

The operators $\mathcal{L}_{0}$ and $\mathcal{L}_{1}$ are introduced for the sake of generality, as they permit to recover a number of particular problems. For instance, taking $\mathcal{L}_{0} u=c^{-2} u, \mathcal{L}_{1} u=0, \mathcal{L}_{2} u=-\Delta u$ and $\delta=0$, we recover the standard Helmholtz equation in an heterogeneous medium with a piecewise smoothly varying sound speed $c \in P C^{\gamma}(\bar{\Omega})$ ( $P C$ meaning that its restriction to $\partial \Omega_{q}$ is in $C^{\gamma, 1}$ ). Other examples are considered and analyzed in the forthcoming sections.

In most applications, system (9) is derived from a time-dependent wave equation by means of Fourier transform. In this case, $\kappa=k$ is real and $\delta=0$. Though some applications employ a complex wave number (obtained from a Laplace transform), we mostly introduce the case where $\delta>0$ as a technicality to analyze problems in which $\Gamma_{D}$ is empty, as depicted in Section 4 .

For further purposes, we need to introduce a new familly of singularities that is related to the splitting of the solution of (9) into a series of power of $k$, that we employ in Theorem 1 . For all $\lambda \in \Lambda_{c}$, we first set $\mathcal{T}_{c,-1}^{\lambda}=\{0\}$ and $\mathcal{T}_{c, 0}^{\lambda}=\mathcal{S}_{c, 0}^{\lambda}$, if $\operatorname{Re} \lambda \in\left(1-\frac{N}{2}, 2-\frac{N}{2}\right)$. Then for all $j \in \mathbb{N}^{*}$ and all $\lambda \in \Lambda_{c}$ such that $\operatorname{Re} \lambda \in\left(1-\frac{N}{2}, j+2-\frac{N}{2}\right)$, we denote by

$\mathcal{T}_{c, j}^{\lambda}=\left\{S^{\lambda} \in \operatorname{span}_{\ell=0}^{j(\lambda)} S_{c}^{\lambda+\ell} ; \quad\right.$ satisfying (7)

or (11) below if $\operatorname{Re} \lambda<j-\frac{N}{2}$ or (12) below if $\left.\operatorname{Re} \lambda<j+1-\frac{N}{2}\right\}$,

$$
\left\{\begin{aligned}
\mathcal{L}_{2} S^{\lambda}-\mathcal{L}_{0} T^{\lambda} & \in P H^{j}(\Omega), & \\
\mathcal{B}_{A} S^{\lambda} & \in P H^{j+\frac{1}{2}}\left(\Gamma_{A}\right), & \\
\mathcal{B}_{D} S^{\lambda} & =0 & \text { on } \Gamma_{D},
\end{aligned}\right.
$$

for some $T^{\lambda} \in \mathcal{T}_{c, j-2}^{\lambda}$

$$
\left\{\begin{array}{rll}
\mathcal{L}_{2} S^{\lambda}-i \mathcal{L}_{1} T^{\lambda} & \in P H^{j}(\Omega), & \\
\mathcal{B}_{A} S^{\lambda}-i A T^{\lambda} & \in P H^{j+\frac{1}{2}}\left(\Gamma_{A}\right), & \\
\mathcal{B}_{D} S^{\lambda} & =0 & \text { on } \Gamma_{D},
\end{array}\right.
$$

for some $T^{\lambda} \in \mathcal{T}_{c, j-1}^{\lambda}$. Before going on, let us point out that

$$
\mathcal{S}_{c, j}^{\lambda} \subset \mathcal{T}_{c, j}^{\lambda} .
$$

The existence of a solution to (11) or (12) is here supposed. In practical examples, it is garanteed by an asymptotic development of $\mathcal{L}_{2}, \mathcal{L}_{1}, \mathcal{L}_{0}$ and $\mathcal{B}_{A}$ at a corner $c$, see Dauge (1988); Dauge et al. (1990) and a logarithmo-polynomial resolution Dauge (1988); Dauge et al. (1990); Nicaise (1993). We refer to Sections 4 and 6 for concrete examples. 
Let us now denote by $\left\{T_{c, j, q}^{\lambda}\right\}_{q=1}^{M_{c, j}^{\lambda}}$ a basis of $\mathcal{T}_{c, j}^{\lambda}$. With the help of these spaces, for any $l \in \mathbb{N} \backslash\{0,1\}$, we introduce the augmented Sobolev space:

$$
A^{l}(\Omega)=P H^{l}(\Omega)^{S} \oplus \operatorname{span}_{c \in \mathcal{C}} \operatorname{span}_{\lambda \in \Lambda_{c} ; \operatorname{Re} \lambda \in\left(1-\frac{N}{2}, l-\frac{N}{2}\right)} \mathcal{T}_{c, l-2}^{\lambda},
$$

which is a Hilbert space with the norm

$$
\|u\|_{A, l, \Omega}^{2}=\left\|u_{\mathrm{reg}}\right\|_{\mathcal{Q}, l, \Omega}^{2}+\sum_{c \in \mathcal{C}} \sum_{\lambda \in \Lambda_{c} ; \operatorname{Re}} \sum_{\lambda \in\left(1-\frac{N}{2}, l-\frac{N}{2}\right)}^{M_{c, l-2}^{\lambda}}\left|c_{c, q}^{\lambda}\right|^{2},
$$

when $u$ admits the splitting

$$
u=u_{\mathrm{reg}}+\sum_{c \in \mathcal{C}} \sum_{\lambda \in \Lambda_{c} ; \operatorname{Re}} \sum_{\lambda \in\left(1-\frac{N}{2}, l-\frac{N}{2}\right)} \sum_{q=1}^{M_{c, l-2}^{\lambda}} c_{c, q}^{\lambda} T_{c, l-2, q}^{\lambda},
$$

where $u_{\text {reg }} \in P H^{l}(\Omega)$ and $c_{c, q}^{\lambda} \in \mathbb{C}$. For shortness we set $A^{0}(\Omega)=L^{2}(\Omega)$ and $A^{1}(\Omega)=H^{1}(\Omega)$.

Definition 2. We will say that system (9) satisfies the k-stability property if it is well-posed in $A^{2}(\Omega)$ for all $k \geq 1$ and if there exists a constant $\alpha \geq 0$ (independent of $k$ ) such that for all $f \in L^{2}(\Omega)^{S}$ the solution $u$ satisfies

$$
k\|u\|_{0, \Omega}+|u|_{1, \Omega}+k^{-1}\|u\|_{A, 2, \Omega} \lesssim k^{\alpha}\|f\|_{0, \Omega},
$$

for all $k \geq 1$.

Remark 2. In the previous definition, the assumption that the stability constant grows algebraically in $k$ is only made for the sake of simplicity. All the results stated below remain valid if a more general stability constant $C_{\text {stab }}(k)$ is employed (assuming that it is bounded for all $k$ but might have a faster growth behavior), by simply replacing $k^{\alpha}$ by $C_{\text {stab }}(k)$.

Before proving our main result, we record a proposition that is very simple, but useful in many places.

Proposition 1. Assume that $\mathcal{L}_{2}$ satisfies the shift property introduced in Definition 1 and that $\tilde{u} \in H^{1}(\Omega)^{S}$ solves

$$
\left\{\begin{aligned}
\mathcal{L}_{2} \tilde{u} & =\mathcal{L}_{0} \tilde{f}+i \mathcal{L}_{1} \tilde{g} & & \text { in } \Omega \\
\mathcal{B}_{A} \tilde{u} & =i A \tilde{g} & & \text { on } \Gamma_{A}, \\
\mathcal{B}_{D} \tilde{u} & =0 & & \text { on } \Gamma_{D},
\end{aligned}\right.
$$

for some $\tilde{f} \in A^{l}(\Omega)$ and $\tilde{g} \in A^{l+1}(\Omega)$ with $l \leq \gamma$. Then $\tilde{u} \in A^{l+2}(\Omega)$ and

$$
\|\tilde{u}\|_{A, l+2, \Omega} \lesssim\|\tilde{f}\|_{A, l, \Omega}+\|\tilde{g}\|_{A, l+1, \Omega},
$$

where the implicit constant depends on $\|A\|_{\mathcal{Q}, \gamma+1, \infty, \Gamma_{A}}$.

Proof. The proof simply relies on shift property (8) and the construction of the spaces $\mathcal{T}_{c, j}^{\lambda}$. Indeed, we first write

$$
\begin{gathered}
\tilde{f}=\tilde{f}_{\mathrm{reg}}+\sum_{c \in \mathcal{C}} \sum_{\lambda \in \Lambda_{c} ; \operatorname{Re}} \sum_{\lambda \in\left(1-\frac{N}{2}, l-\frac{N}{2}\right)} \sum_{q=1}^{M_{c, l-2}^{\lambda}} c_{c, q}^{\lambda} T_{c, l-2, q}^{\lambda}, \\
\tilde{g}=\tilde{g}_{\mathrm{reg}}+\sum_{c \in \mathcal{C}} \sum_{\mu \in \Lambda_{c} ; \operatorname{Re} \mu \in\left(1-\frac{N}{2}, l+1-\frac{N}{2}\right)} \sum_{q^{\prime}=1}^{M_{c, l-1}^{\mu}} d_{c, q}^{\mu} T_{c, l-1, q^{\prime}}^{\mu},
\end{gathered}
$$

when $\tilde{f}_{\text {reg }} \in P H^{l}(\Omega), \tilde{g}_{\text {reg }} \in P H^{l+1}(\Omega)$, and $c_{c, q}^{\lambda}, d_{c, q}^{\mu} \in \mathbb{C}$. Then by the definition of $\mathcal{T}_{c, l}^{\lambda}$ and $\mathcal{T}_{c, l}^{\mu}$, for all $\lambda \in \Lambda_{c} ; \operatorname{Re} \lambda \in\left(1-\frac{N}{2}, l-\frac{N}{2}\right), q=1, \cdots, M_{c, l-2}^{\lambda}$ and $\mu \in \Lambda_{c} ; \operatorname{Re} \mu \in\left(1-\frac{N}{2}, l+1-\frac{N}{2}\right)$, 
$q^{\prime}=1, \cdots, M_{c, l-1}^{\mu}$, there exist $S_{c, q, 1}^{\lambda} \in \mathcal{T}_{c, l}^{\lambda}$ and $S_{c, q^{\prime}, 0}^{\mu} \in \mathcal{T}_{c, l}^{\mu}$ solution of (compare with (11) and $(12))$

$$
\left\{\begin{array}{rlr}
\mathcal{L}_{2} S_{c, q, 0}^{\lambda}-\mathcal{L}_{0} T_{c, q}^{\lambda} & \in P H^{l}(\Omega), & \\
\mathcal{B}_{A} S_{c, q, 0}^{\lambda} & \in P H^{l+\frac{1}{2}}\left(\Gamma_{A}\right), & \\
\mathcal{B}_{D} S_{c, q, 0}^{\lambda} & =0 & \text { on } \Gamma_{D}
\end{array}\right.
$$

and

$$
\left\{\begin{aligned}
\mathcal{L}_{2} S_{c, q^{\prime}, 1}^{\mu}-i \mathcal{L}_{1} T_{c, q^{\prime}}^{\mu} & \in P H^{l}(\Omega), & \\
\mathcal{B}_{A} S_{c, q^{\prime}, 1}^{\mu}-i A T_{c, q^{\prime}}^{\mu} & \in P H^{l+\frac{1}{2}}\left(\Gamma_{A}\right), & \\
\mathcal{B}_{D} S_{c, q^{\prime}, 1}^{\mu} & =0 & \text { on } \Gamma_{D} .
\end{aligned}\right.
$$

Consequently the function

$$
\begin{aligned}
U & =\tilde{u}-\sum_{c \in \mathcal{C}} \sum_{\lambda \in \Lambda_{c} ; \operatorname{Re}} \sum_{\lambda \in\left(1-\frac{N}{2}, l-\frac{N}{2}\right)} \sum_{q=1}^{M_{c, l-2}^{\lambda}} c_{c, q}^{\lambda} S_{c, q, 0}^{\lambda} \\
& -\sum_{c \in \mathcal{C}} \sum_{\mu \in \Lambda_{c} ; \operatorname{Re}} \sum_{\mu \in\left(1-\frac{N}{2}, l+1-\frac{N}{2}\right)} \sum_{q^{\prime}=1}^{M_{c, l-1}^{\mu}} d_{c, q}^{\mu} S_{c, q^{\prime}, 1}^{\mu},
\end{aligned}
$$

is solution of

$$
\left\{\begin{aligned}
\mathcal{L}_{2} U & =F & & \text { in } \Omega, \\
\mathcal{B}_{A} U & =i A G & & \text { on } \Gamma_{A}, \\
\mathcal{B}_{D} U & =0 & & \text { on } \Gamma_{D},
\end{aligned}\right.
$$

with $F \in P H^{l}(\Omega)$ and $G \in P H^{l+1 / 2}\left(\Gamma_{A}\right)$ such that (using a trace theorem and the fact that $A$ does not depend on $k$ )

$$
\|F\|_{\mathcal{Q}, l, \Omega}+\|G\|_{\mathcal{Q}, l+1 / 2, \Gamma_{A}} \lesssim\|\tilde{f}\|_{A, l, \Omega}+\|\tilde{g}\|_{A, l+1, \Omega} .
$$

Hence using the shift property (1), we deduce that $U \in A^{l+2}(\Omega)$ with

$$
\|U\|_{A, l+2, \Omega} \lesssim\|F\|_{\mathcal{Q}, l, \Omega}+\|G\|_{\mathcal{Q}, l+1 / 2, \Gamma_{A}} .
$$

The last two estimates and the definition of $U$ allows to conclude.

We are now ready to establish the main result of this section.

Theorem 1. Assume that the assumptions of Definitions 1 and 2 are fulfilled. For $f \in L^{2}(\Omega)^{S}$, define the sequence $u_{j}$ by

$$
\begin{gathered}
\left\{\begin{array}{rll}
\mathcal{L}_{2} u_{0}=f & \text { in } \Omega, \\
\mathcal{B}_{A} u_{0}=0 & \text { on } \Gamma_{A}, \\
\mathcal{B}_{D} u_{0}=0 & \text { on } \Gamma_{D},
\end{array}\right. \\
\begin{cases}\mathcal{L}_{2} u_{1}=i \mathcal{L}_{1} u_{0} & \text { in } \Omega, \\
\mathcal{B}_{A} u_{1}=i A u_{0} & \text { on } \Gamma_{A}, \\
\mathcal{B}_{D} u_{1}=0 & \text { on } \Gamma_{D},\end{cases}
\end{gathered}
$$

and

$$
\left\{\begin{aligned}
\mathcal{L}_{2} u_{j} & =\mathcal{L}_{0} u_{j-2}+i \mathcal{L}_{1} u_{j-1} & & \text { in } \Omega, \\
\mathcal{B}_{A} u_{j} & =i A u_{j-1} & & \text { on } \Gamma_{A}, \\
\mathcal{B}_{D} u_{j} & =0 & & \text { on } \Gamma_{D}
\end{aligned}\right.
$$

for $j \geq 2$.

Then, we have $u_{j} \in A^{j+2}(\Omega)$ with

$$
\left\|u_{j}\right\|_{A, j+2, \Omega} \lesssim\|f\|_{0, \Omega},
$$

for $0 \leq j \leq \gamma$. Furthermore, for any $l \leq \gamma$, if we denote by

$$
r_{l}=u-\sum_{j=0}^{l-1} \kappa^{j} u_{j}
$$


where $u$ is the solution to system (9) associated with $f$, then $r_{l} \in A^{l+2}(\Omega)$ with

$$
\left\|r_{l}\right\|_{A, l+2, \Omega} \lesssim k^{l+1+\alpha}\|f\|_{0, \Omega} .
$$

Remark 3. The definition of the sequence $u_{j}$ is based on formal expansion

$$
u=\sum_{j=0}^{+\infty} \kappa^{j} u_{j}
$$

in powers of $\kappa$. The recursive definition of the $u_{j}$ in terms of $u_{j-1}$ and $u_{j-2}$ is obtained by injecting formal expansion (20) into boundary value problem (9) and identifying powers of $\kappa$.

Remark 4. Theorem 1 defines the announced splitting of the solution $u$. Indeed, we have $u=$ $u_{0}+r_{l}$ with

$$
u_{0}=\sum_{j=0}^{l-1} \kappa^{j} u_{j}
$$

We see that $u_{0}$ only belongs to $A^{2}(\Omega)$, while $r_{l} \in A^{l+2}(\Omega)$. $u_{0}$ thus corresponds to a "rough" component of the solution, whose norm is efficiently controlled in terms of $k$. On the other hand, $r_{l}$ is "smooth" but its norm strongly increases with $k$.

Remark 5. If we consider subdomains with smooth boundaries of class $C^{\gamma+1,1}$, we can simply replace $A^{l}(\Omega)$ by $\mathrm{PH}^{l}(\Omega)^{S}$ in the statement of Theorem 1.

Proof. The fact that each $u_{j} \in A^{j+2}(\Omega)$ directly follows by applying shift property (14) recursively in the definition of the sequence. It is also clear that (18) holds, since $k$ is not involved in the definition of the sequence.

Simple computations show that the sequence $r_{l}$, for $1 \leq l \leq \gamma$ recursively satisfies

$$
\begin{aligned}
& \left\{\begin{array}{lll}
\mathcal{L}_{2} r_{1}=\kappa^{2} \mathcal{L}_{0} u+i \kappa \mathcal{L}_{1} u & & \text { in } \Omega, \\
\mathcal{B}_{A} r_{1}=i \kappa A u & & \text { on } \Gamma_{A}, \\
\mathcal{B}_{D} r_{1}=0 & & \text { on } \Gamma_{D},
\end{array}\right. \\
& \left\{\begin{aligned}
\mathcal{L}_{2} r_{2} & =\kappa^{2} \mathcal{L}_{0} u+i \kappa \mathcal{L}_{1} r_{1} & & \text { in } \Omega, \\
\mathcal{B}_{A} r_{2} & =i \kappa A r_{1} & & \text { on } \Gamma_{A}, \\
\mathcal{B}_{D} r_{2} & =0 & & \text { on } \Gamma_{D},
\end{aligned}\right.
\end{aligned}
$$

and

$$
\left\{\begin{aligned}
\mathcal{L}_{2} r_{l} & =\kappa^{2} \mathcal{L}_{0} r_{l-2}+i \kappa \mathcal{L}_{1} r_{l-1} & & \text { in } \Omega \\
\mathcal{B}_{A} r_{l} & =i \kappa A r_{l-1} & & \text { on } \Gamma_{A}, \\
\mathcal{B}_{D} r_{l} & =0 & & \text { on } \Gamma_{D}
\end{aligned}\right.
$$

for $l \geq 3$.

Hence we apply shift property (14) to system (21), so that

$$
\left\|r_{1}\right\|_{A, 3, \Omega} \lesssim k^{2}\|u\|_{1, \Omega}+k\|u\|_{A, 2, \Omega} .
$$

Then, as a result, we obtain

$$
\left\|r_{1}\right\|_{A, 3, \Omega} \lesssim k^{2+\alpha}\|f\|_{0, \Omega}
$$

by using (13). We thus have proved (19) for $l=1$.

The case $l=2$ also follows with the use of (13) and (14). Indeed, since we already proved (24), we have

$$
\left\|r_{2}\right\|_{A, 2, \Omega} \lesssim k^{2}\|u\|_{A, 2, \Omega}+k\left\|r_{1}\right\|_{A, 3, \Omega} \lesssim k^{3+\alpha}\|f\|_{0, \Omega} .
$$

Estimate (19) being established for the case $l=1$ and 2 . We can obtain the general case by induction. Thus, assume that (19) holds up to some $n-1$ with $3 \leq n \leq \gamma$. Applying shift property (14) to (23) and the inductive assumption, we have

$$
\left\|r_{n}\right\|_{A, n+2, \Omega} \lesssim k^{2}\left\|r_{n-2}\right\|_{A, n, \Omega}+k\left\|r_{n-1}\right\|_{A, n+1, \Omega} \lesssim k^{n+1+\alpha}\|f\|_{0, \Omega},
$$

which is (19) for $l=n$. 
2.2. Application to finite element discretizations. We take advantage of the splitting proposed in Theorem 1 to derive stability conditions and error-estimates for finite element discretizations of system (9).

We recall the variational setting of problem (9). For that purpose, we introduce the space

$$
H_{\Gamma_{D}}^{1}(\Omega)=\left\{v \in H^{1}(\Omega) \mid \mathcal{B}_{D} v=0 \text { on } \Gamma_{D}\right\},
$$

equipped with the norm of $H^{1}(\Omega)$. As $\delta$ is fixed, for each $k \geq 1$, we consider a sesquilinear form $b_{k}: H_{\Gamma_{D}}^{1}(\Omega)^{S} \times H_{\Gamma_{D}}^{1}(\Omega)^{S} \rightarrow \mathbb{C}$ that represents a variational formulation of $(9)$, so that its solution is characterized as

$$
b_{k}(u, v)=(f, v), \quad \forall v \in H_{\Gamma_{D}}^{1}(\Omega)^{S} .
$$

Let us denote by $\mathcal{P}_{k}$ and $\mathcal{P}_{k}^{\star}$ the linear operators from $H_{\Gamma_{D}}^{1}(\Omega)^{S}$ to $\left(H_{\Gamma_{D}}^{1}(\Omega)^{S}\right)^{\prime}$ defined by

$$
\left\langle\mathcal{P}_{k} u, v\right\rangle_{-1,1}=b_{k}(u, v)=\left\langle u, \mathcal{P}_{k}^{\star} v\right\rangle_{1,-1}, \forall u, v \in H_{\Gamma_{D}}^{1}(\Omega)^{S},
$$

where the duality pairing $\langle\cdot, \cdot\rangle_{-1,1}\left(\operatorname{resp} .\langle\cdot, \cdot\rangle_{1,-1}\right)$ means the one between $\left(H_{\Gamma_{D}}^{1}(\Omega)^{S}\right)^{\prime}$ and $H_{\Gamma_{D}}^{1}(\Omega)^{S}$ (resp. $H_{\Gamma_{D}}^{1}(\Omega)^{S}$ and $\left.\left(H_{\Gamma_{D}}^{1}(\Omega)^{S}\right)^{\prime}\right)$.

Throughout this section, it will be convenient to consider the weighted norm

$$
\|v\|\left\|^{2}=k^{2}\right\| v \|_{0, \Omega}^{2}+|v|_{1, \Omega}^{2}, \quad v \in H^{1}(\Omega),
$$

that is equivalent to the natural $H^{1}(\Omega)$ norm. In view of $(13)$, the ||$|\cdot|||$-norm has the advantage to "balance" the $L^{2}$ and $H^{1}$ terms of the $H^{1}(\Omega)$ norm.

Assumption 1. We will assume that the sesquilinear form $b_{k}$ is continuous, in the sense that

$$
\left|b_{k}(u, v)\right| \lesssim\||u|\| \cdot\||v|\|, \quad \forall u, v \in H_{\Gamma_{D}}^{1}(\Omega)^{S},
$$

and satisfies the Gårding inequality

$$
\operatorname{Re} b_{k}(u, u) \gtrsim|u|_{1, \Omega}^{2}-k^{2}\|u\|_{0, \Omega}^{2}, \quad \forall u \in H_{\Gamma_{D}}^{1}(\Omega)^{S} .
$$

We look for a finite element approximation $u_{h, p}$ to $u$. To this end, we consider a family of meshes $\left\{\mathcal{T}_{h}\right\}_{h}$ of $\Omega$, where each mesh is made of tetrahedral (or triangular) elements $K$. To simplify the analysis, we assume that the boundary of $\Omega$ is exactly triangulated, and therefore, we consider curved Lagrange finite elements as described in Bernardi (1989). Also, for each element $K$, we denote by $\mathcal{F}_{K}$ the mapping taking the reference element $\hat{K}$ to $K$. We further assume that the triangulation is conform with the partition $\mathcal{Q}$, in other words, each element $K \in \mathcal{T}_{h}$ is supposed to be included into one and only one $\overline{\Omega_{q}}$.

Then, for all $p \leq \gamma+1$, the finite element approximation space $V_{h, p}$ is defined as

$$
V_{h, p}=\left\{v_{h, p} \in H_{\Gamma_{D}}^{1}(\Omega)^{S}\left|v_{h, p}\right|_{K} \circ \mathcal{F}_{K}^{-1} \in \mathbb{P}_{p}(\hat{K})^{S} \forall K \in \mathcal{T}_{h}\right\}
$$

where $\mathbb{P}_{p}(\hat{K})$ stands for the set of polynomials of total degree less than or equal to $p$. At that point, a finite element approximation of $u$ is obtained by looking for $u_{h, p} \in V_{h, p}$ such that

$$
b_{k}\left(u_{h, p}, v_{h, p}\right)=\left(f, v_{h, p}\right), \quad \forall v_{h, p} \in V_{h, p} .
$$

Remark 6. In actual applications, exact triangulations are seldom used, as they are difficult to build (see $\S 6$ of Bernardi (1989) in particular). Instead, isoparametric meshes, in which the non-affine mappings $\mathcal{F}_{K}$ are assumed to be a polynomial mapping of order $p$ are usually preferred as they deliver the same orders of convergence. However, the resulting finite element spaces are non-conforming, since the boundary conditions can not be exactly embedded. For the sake of simplicity, we shall focus on the case of exact triangulations with general element mappings, and omit the analysis of the "variational crime" caused by isoparametric elements. The authors believe that similar results as presented here can be obtained for isoparametric elements, at the price of additional complexities in the proofs.

In the rest of this section, we only consider meshes that have "sufficiently many" elements per wavelength. We thus make the assumption that

$$
k h \lesssim 1 .
$$


This assumption is fairly general, and only rules out meshes that would produce inaccurate solutions. As a result, it is usually satisfied in applications. In addition, the convergence analysis leads to conditions on $h$ that are actually more restrictive than (28).

We assume that the considered family of meshes is regular of order $p$ in the sense given in Bernardi (1989). Roughly speaking, it consists in assuming that each element $K$ of each mesh $\mathcal{T}_{h}$ is obtained from a single reference elements through a mapping $\mathcal{F}_{K}$ that is a "small perturbation" of an affine mapping $\tilde{F}_{K}$. In addition, the "perturbations" are assumed to be smooth, and satisfy some bounds on their gradient uniformly in $h$. In case of singular corners, we further need to refine the meshes near such corners, see for instance Babuška (1970); Lubuma \& Nicaise (1995). For simplicity, for each corner $c \in \mathcal{C}$, we suppose that ${ }^{1}$

$$
\frac{N}{2}+\operatorname{Re} \lambda \notin \mathbb{Z}, \forall \lambda \in \Lambda_{c} .
$$

$\lambda_{\min }$ as the singular exponent from $\cup_{c \in \mathcal{C}} \Lambda_{c}$ satisfying

$$
\operatorname{Re} \lambda_{\min }=\min \left\{\operatorname{Re} \lambda ; \lambda \in \cup_{c \in \mathcal{C}} \Lambda_{c} \text { and } \operatorname{Re} \lambda>1-\frac{N}{2}\right\},
$$

and suppose in dimension 3 that $^{2}$

$$
\operatorname{Re} \lambda_{\min }>0
$$

Let us then define $l^{*} \in \mathbb{N}^{*}$ as the unique integer such that

$$
l^{*}-\frac{N}{2}<\operatorname{Re} \lambda_{\min }<l^{*}+1-\frac{N}{2} .
$$

Then for all $l \geq l^{*}$, we introduce the following mesh refinement conditions, see Babuška (1970); Lubuma \& Nicaise (1995):

$$
\begin{aligned}
& h_{K} \lesssim h^{l /\left(l-\alpha_{l}\right)}, \text { if } K \text { contains a corner, } \\
& h_{K} \lesssim h \inf _{K} r^{\alpha_{l} / l}, \text { otherwise, }
\end{aligned}
$$

where $r=\min _{c \in \mathcal{C}} r_{c}$ is the distance to the corners, $\alpha_{l}=\alpha_{l}^{\star}+l-l^{*}$, and $\alpha_{l}^{\star}$ is a fixed positive real number satisfying

$$
l^{*}+1-\frac{N}{2}-\operatorname{Re} \lambda_{\min }<\alpha_{l}^{\star}<l^{*}-1 .
$$

In the following we assume that the family of meshes is regular and that for each $h>0$, conditions (31)-(32) are satisfied by all elements $K \in \mathcal{T}_{h} .{ }^{3}$ Under such assumptions the next interpolation error estimates are valid.

Lemma 1. For each $v \in A^{l+1}(\Omega) \cap H_{\Gamma_{D}}^{1}(\Omega)^{S}(1 \leq l \leq p)$, if the family of meshes is regular and satisfies (31)-(32) for $l \geq l^{*}$, then there exists an element $\mathcal{I}_{h, p} v \in V_{h, p}$ such that

$$
\left|v-\mathcal{I}_{h, p} v\right|_{j, \Omega} \lesssim h^{l+1-j}\|v\|_{A, l+1, \Omega}, \quad(0 \leq j \leq 1) .
$$

Proof. According to Babuška (1970); Lubuma \& Nicaise (1995), for all $1 \leq l \leq p$, we introduce the space

$$
P H^{l+1, \alpha_{l}}(\Omega)=\left\{u \in H^{1}(\Omega) ; r^{\alpha_{l}} D^{\beta} u_{\mid \Omega_{q}} \in L^{2}\left(\Omega_{q}\right), \forall \beta \in \mathbb{N}^{N}: 2 \leq|\beta| \leq l+1, p \in \mathcal{Q}\right\},
$$

that is an Hilbert space with its natural norm, where $\alpha_{l}=0$ if $l<l^{*}$.

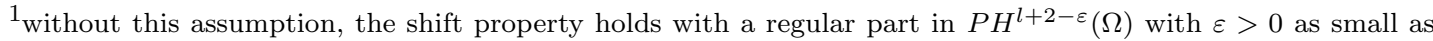
we want, leading to a lose of a factor $h^{\varepsilon}$ in the rate of convergence. For simplicity, this exceptional situation will be not explicitely mentioned in our examples.

${ }^{2}$ This condition is satisfied in the case of the acoustic Helmholtz equation in homogeneous media and in the case of the time-harmonic elastodynamic system, see Remarks 8 and 10. In addition, this assumption is not a fundamental limitation of our analysis, but is a technicality that is required to properly define the Lagrange interpolant later on. It is probably possible to remove such hypothesis by employing quasi-interpolation operators.

3 as the singularities are local, the refinement conditions can be defined locally near each corner with different grading conditions, we avoid this specification to lighten the presentation Let us point out that in the case of smooth $C^{\gamma+1,1}$ subdomains $\Omega_{q}$, it is not required to perform any kind of refinement.
} 
Now combining the arguments from Theorem 3.3 of Lubuma \& Nicaise (1995) (let us notice that the assumption on $\alpha_{l}$ yields that $P H^{l+1, \alpha_{l}}(\Omega)$ is continuously embedded into $P H^{N / 2+\varepsilon}(\Omega)$, for some $\varepsilon>0$ ) with the ones from Bernardi (1989), one can show that

$$
\left|v-\mathcal{I}_{h, p} v\right|_{j, \Omega} \lesssim h^{l+1-j}\|v\|_{P H^{l+1, \alpha_{l}(\Omega)}}, \quad(0 \leq j \leq 1),
$$

where $\mathcal{I}_{h, p} v$ is the Lagrange interpolant of $v$.

As the assumption (30) and simple calculations allow to show that $A^{l+1}(\Omega)$ is continuously embedded into $P H^{l+1, \alpha_{l}}(\Omega)^{S}$, the conclusion follows.

Proposition 2. Assume that $v \in A^{l+1}(\Omega)$ with $1 \leq l \leq p$, and that the family of meshes is regular and satisfies (31)-(32) for $l \geq l^{*}$, then there exists an element $\mathcal{I}_{h, p} v \in V_{h, p}$ such that

$$
\left\|\left|\left\|v-\mathcal{I}_{h, p} v \mid\right\| \lesssim h^{l}\|v\|_{A, l+1, \Omega} .\right.\right.
$$

Proof. The proof is a direct consequence of the assumption that $k h \lesssim 1$ and estimate (34). Indeed, we have

$$
\begin{aligned}
\left\|v-\mathcal{I}_{h, p} v\right\| \| & \lesssim k\left\|v-\mathcal{I}_{h, p} v\right\|_{0, \Omega}+\left|v-\mathcal{I}_{h, p} v\right|_{1, \Omega} \\
& \lesssim k h^{l+1}\|v\|_{A, l+1, \Omega}+h^{l}\|v\|_{A, l+1, \Omega} \\
& \lesssim(1+k h) h^{l}\|v\|_{A, l+1, \Omega} .
\end{aligned}
$$

The main result of this section is an asymptotic error estimate for the finite element solution $u_{h, p}$ that is explicit in terms of $h, p$ and $k$. The main steps to obtain this estimate rely on slight modifications of the so-called Schatz argument (Schatz, 1974), as presented in Melenk \& Sauter (2010) and Melenk \& Sauter (2011) for the acoustic Helmholtz equation. For the sake of completeness, we briefly reproduce this argument with slight variations for the case of our general settings in Lemmas 2 and 3.

Lemma 2. Assume that the boundary value problem associated with the operator $\mathcal{P}_{k}^{\star}$ can be decomposed into system (9) in such a way that the assumptions from Definitions 1 and 2 hold. Then, for $g \in L^{2}(\Omega)^{S}$, there exists a unique $s^{\star}(g) \in H_{\Gamma_{D}}^{1}(\Omega)^{S}$ such that

$$
b_{k}\left(\phi, s^{\star}(g)\right)=(\phi, g), \quad \forall \phi \in H_{\Gamma_{D}}^{1}(\Omega)^{S} .
$$

Furthermore, if we denote by

$$
\eta_{h, p}=\sup _{g \in L^{2}(\Omega)} \frac{\left\|s^{\star}(g)-\mathcal{I}_{h, p} s^{\star}(g)\right\| \mid}{\|g\|_{0, \Omega}},
$$

and if the family of meshes is regular and satisfies (31)-(32) for $p \geq l^{*}$, then

$$
\eta_{h, p} \lesssim h+k^{p+\alpha} h^{p}
$$

Proof. Let $g \in L^{2}(\Omega)^{S}$, the existence and uniqueness of $s^{\star}(g)$ directly follows since $\mathcal{P}_{k}^{\star}$ satisfies the assumptions from Definition 2. In addition, we see that $s^{\star}(g)$ can be split up using the technique introduced in the previous section. In other words, we have

$$
s^{\star}(g)=\sum_{j=0}^{p-2} \kappa^{j} s_{j}+r_{p-1},
$$

where $s_{j} \in A^{j+2}(\Omega), r_{p-1} \in A^{p+1}(\Omega)$, and

$$
\left\|s_{j}\right\|_{A, j+2, \Omega} \lesssim\|g\|_{0, \Omega}, \quad\left\|r_{p-1}\right\|_{A, p+1, \Omega} \lesssim k^{p+\alpha}\|g\|_{0, \Omega} .
$$


Then, using (35) and recalling the assumption that $k h \lesssim 1$, we have

$$
\begin{aligned}
\left\|s^{\star}(g)-\mathcal{I}_{h} s^{\star}(g)\right\| \| & \lesssim \sum_{j=0}^{p-2} k^{j}\left\|\mid s_{j}-\mathcal{I}_{h} s_{j}\right\|+\left\|r_{p-1}-\mathcal{I}_{h} r_{p-1}\right\| \| \\
& \lesssim \sum_{j=0}^{p-2} h^{j+1} k^{j}\left\|s_{j}\right\|_{A, j+2, \Omega}+h^{p}\left\|r_{p-1}\right\|_{A, p+1, \Omega} \\
& \lesssim \sum_{j=0}^{p-2} h(h k)^{j}\left\|s_{j}\right\|_{A, j+2, \Omega}+h^{p}\left\|r_{p-1}\right\|_{A, p+1, \Omega} \\
& \lesssim \sum_{j=0}^{p-2} h\left\|s_{j}\right\|_{A, j+2, \Omega}+h^{p}\left\|r_{p-1}\right\|_{A, p+1, \Omega} \\
& \lesssim\left(h+k^{p+\alpha} h^{p}\right)\|g\|_{0, \Omega},
\end{aligned}
$$

and the result follows.

Lemma 3. Assume that $b_{k}$ satisfies Assumption 1. Then, if $k \eta_{h, p}$ is small enough, there exists a unique finite element solution $u_{h, p} \in V_{h, p}$ to (27), and we have

$$
\left\|\mid u-u_{h, p}\right\|\left\|\lesssim \inf _{\phi_{h, p} \in V_{h, p}}\right\| u-\phi_{h, p}\|\| .
$$

Proof. Using (26), there exists a positive constant $M$ independent of $k$ and $h$ such that

$$
\begin{aligned}
\operatorname{Re} b_{k}\left(u-u_{h, p}, u-u_{h, p}\right) & \geq M\left(-k^{2}\left\|u-u_{h, p}\right\|_{0, \Omega}^{2}+\left|u-u_{h, p}\right|_{1, \Omega}^{2}\right) \\
& \geq M \mid\left\|u-u_{h, p}\right\|^{2}-2 M k^{2}\left\|u-u_{h, p}\right\|_{0, \Omega}^{2} .
\end{aligned}
$$

If we define $\xi=2 M k^{2} s^{\star}\left(u-u_{h, p}\right)$, we have

$$
\left\|u-u_{h, p} \mid\right\|^{2} \lesssim \operatorname{Re} b_{k}\left(u-u_{h, p}, u-u_{h, p}+\xi\right) .
$$

Then, by Galerkin orthogonality, we can replace $u_{h, p}$ by $\phi_{h, p}+\mu_{h, p}$ for arbitrary $\phi_{h, p}, \mu_{h, p} \in V_{h, p}$, and it follows that

$$
\begin{aligned}
\operatorname{Re} b_{k}\left(u-u_{h, p}, u-u_{h, p}+\xi\right) & =\operatorname{Re} b_{k}\left(u-u_{h, p}, u-\phi_{h, p}\right) \\
& +\operatorname{Re} b_{k}\left(u-u_{h, p}, \xi-\mu_{h, p}\right) \\
& \lesssim\left\|| \| - u _ { h , p } \| | \left(\left\||| u-\phi_{h, p}||+||\left|\xi-\mu_{h, p} \|\right|\right),\right.\right.
\end{aligned}
$$

where we have used (25). As (36) holds for arbitrary $\phi_{h, p}, \mu_{h, p} \in V_{h, p}$, we can take the infinimum in the right hand side. In addition, by definition of $\xi$ and $\eta_{h, p}$, we have that

$$
\inf _{\mu_{h, p} \in V_{h, p}}\left\|\left|\xi-\mu_{h, p}\left\|\left|\lesssim k^{2} \eta_{h, p}\left\|u-u_{h, p}\right\|_{0, \Omega} \lesssim k \eta_{h, p}\left\|\mid u-u_{h, p}\right\| \| .\right.\right.\right.\right.
$$

We thus obtain that

$$
\left\|u-u_{h, p} \mid\right\| \leq \rho\left(\operatorname { i n f } _ { \phi _ { h , p } \in V _ { h , p } } \| u - \phi _ { h , p } \| \left|+k \eta_{h, p}\left\|\left|u-u_{h, p} \|\right|\right),\right.\right.
$$

where $\rho$ is a constant independent of $h$ and $k$, which we can rewrite as

$$
\left(1-k \eta_{h, p} \rho\right)\left|\left\|u-u_{h, p} \mid\right\| \lesssim \inf _{\phi_{h, p} \in V_{h, p}}\left\|u-\phi_{h, p}\right\| \| .\right.
$$

Hence, the main result follows assuming that

$$
k \eta_{h, p} \leq \frac{1}{2 \rho}
$$

We obtain an asymptotic error estimate as a direct consequence of Lemmas 2 and 3. 
Theorem 2. Assume that $\mathcal{P}_{k}^{\star}$ satisfies the assumptions of Definition 1 , that $\mathcal{P}_{k}$ and $\mathcal{P}_{k}^{\star}$ satisfy the assumptions of Definition 2, and that $b_{k}$ satisfies Assumption 1. Let $f \in L^{2}(\Omega)^{S}$, then, if $k h$ and $k^{p+\alpha+1} h^{p}$ are small enough with $p \leq \gamma+1$, and if the family of meshes is regular and satisfies (31)-(32) for $p \geq l^{*}$, there exists a unique finite element solution $u_{h, p} \in V_{h, p}$ to (27), and it holds that

$$
\left\|u-u_{h, p}\right\|\left|\lesssim \inf _{\phi_{h, p} \in V_{h, p}}\left\|u-\phi_{h, p}\right\|\right|
$$

Furthermore, we have that

$$
\left\|\left|u-u_{h, p}\right|\right\| \lesssim k^{1+\alpha} h\|f\|_{0, \Omega} .
$$

In addition, if we assume that $f \in P H^{p-1}(\Omega)^{S}$, then we have

$$
\left\|\left|u-u_{h, p}\left\|\mid \lesssim k^{p+\alpha} h^{p}\right\| f \|_{\mathcal{Q}, p-1, \Omega} .\right.\right.
$$

Remark 7. Stability estimate (37) is still valid if the assumption on the right-hand-side $f \in$ $L^{2}(\Omega)^{S}$ is weaken to $f \in\left(H_{\Gamma_{D}}^{1}(\Omega)^{S}\right)^{\prime}$, however, error estimates (38) and (39) do not hold in this case.

Proof. We obtain (37) as a direct consequence of Lemmas 2 and 3. Then, we deduce (38) from (37) by combining Proposition 2 and Definition 2:

$$
\left|\left\|u-u_{h, p}|||\lesssim||| u-\left.\mathcal{I}_{h, p} u|||\lesssim h| u\right|_{A, 2, \Omega} \lesssim k^{1+\alpha} h\right\| f \|_{0, \Omega} .\right.
$$

In order to prove (39), we first establish by induction that $u \in A^{l+2}(\Omega)$ with

$$
\|u\|_{A, l+2, \Omega} \lesssim k^{l+1+\alpha}\|f\|_{\mathcal{Q}, l, \Omega},
$$

for all $0 \leq l \leq \gamma$. Because of Definition 2, (40) is valid for $l=0$. Let us thus assume that (40) is valid for some $0 \leq l \leq \gamma-1$. Since $u$ solves (9), we have

$$
\left\{\begin{aligned}
\mathcal{L}_{2} u & =f+\kappa^{2} \mathcal{L}_{0} u+i \kappa \mathcal{L}_{1} u & & \text { in } \Omega \\
\mathcal{B}_{A} u & =i \kappa A u & & \text { on } \partial \Omega
\end{aligned}\right.
$$

where $f \in P H^{l+1}(\Omega)^{S}$ and $u \in A^{l+2}(\Omega)$. Thus, the shift property (8) and Propositin 1 imply that $u \in A^{l+3}(\Omega)$ with

$$
\|u\|_{A, l+3, \Omega} \lesssim\|f\|_{\mathcal{Q}, l+1, \Omega}+k^{2}\|u\|_{A, l+1, \Omega}+k\|u\|_{A, l+2, \Omega} .
$$

Hence by the induction hypothesis, we find that

$$
\|u\|_{l+3, \Omega} \lesssim k^{l+2+\alpha}\|f\|_{\mathcal{Q}, l+1, \Omega},
$$

so that (40) is valid for $l+1$. Thus (40) is valid for $0 \leq l \leq \gamma$ by induction. In particular, we have

$$
||\left|u-\mathcal{I}_{h, p} u\right|\left\|\lesssim h^{p}|u|_{A, p+1, \Omega} \lesssim k^{p+\alpha} h^{p}\right\| f \|_{\mathcal{Q}, p-1, \Omega}
$$

for all $p \leq \gamma+1$, and (39) follows from (37).

\section{Application to the acoustic Helmholtz equation in homogeneous media}

The aim of this section is to apply our general result to the simple problem of the acoustic Helmholtz equation in an homogeneous medium. We consider a domain $\Omega \subset \mathbb{R}^{N}$ whose boundary $\partial \Omega$ is split into two subsets $\Gamma_{D}$ and $\Gamma_{A}$, and consider the problem to find $u: \Omega \rightarrow \mathbb{C}$ such that

$$
\left\{\begin{aligned}
-k^{2} u-\Delta u & =f & & \text { in } \Omega, \\
u & =0 & & \text { on } \Gamma_{D}, \\
\nabla u \cdot n-i k u & = & & \text { on } \Gamma_{A},
\end{aligned}\right.
$$

where $f \in L^{2}(\Omega)$ is a given load term. The variational formulation of Problem (42) reads: find $u \in H_{\Gamma_{D}}^{1}(\Omega)$ such that $b_{k}(u, v)=(f, v)$ for all $v \in H_{\Gamma_{D}}^{1}(\Omega)$ where $b_{k}$ takes here the form

$$
b_{k}(u, v)=-k^{2}(u, v)-i k\langle u, v\rangle_{\Gamma_{A}}+(\nabla u, \nabla v) .
$$

Following Hetmaniuk (2007) we assume that there exists a point $x_{0} \in \mathbb{R}^{N}$ such that $\left(x-x_{0}\right)$. $n(x) \leq 0$ for all $x \in \Gamma_{D}$ and $\left(x-x_{0}\right) \cdot n(x) \geq c>0$ for all $x \in \Gamma_{A}$, where $n$ is the unit vector 
normal to $\partial \Omega$ pointing outside $\Omega$. We further assume that $\Gamma_{A}$ and $\Gamma_{D}$ are disjoint, and either that they are of class $C^{\gamma+1,1}$ for some $\gamma \in \mathbb{N}$, or that $N=2$ and that they are polygonal.

We point out that this situation represents in particular the case of the exterior problem outside a star-shaped sound-soft obstacle $\mathcal{O}$ with boundary $\partial \mathcal{O}=\Gamma_{D}$. In this context, the boundary condition placed on $\Gamma_{A}$ is an approximation of the Sommerfeld radiation condition.

Problem (42) enters into our framework with $\mathcal{L}_{0}=I d, \mathcal{L}_{1}=0, \mathcal{L}_{2}=-\Delta, \mathcal{B}_{D}=I d, \mathcal{B}_{A} u=\nabla u \cdot n$ and $A=1$. Furthermore as the operators have constant coefficients, $\mathcal{Q}$ is reduced to a singleton and $P H^{l}(\Omega)=H^{l}(\Omega)$. Since the triplet $\left(\mathcal{L}_{2}, \mathcal{B}_{D}, \mathcal{B}_{A}\right)$ corresponds to the standard Laplace operator with mixed boundary conditions, standard arguments based on elliptic regularity show that the shift property of Definition 1 is satisfied up to the order $\gamma+2$ without singularities for the case of smooth boundaries. When the boundaries are polygonal, the shift property holds for any order, and the set of singular functions can be explicitly described (see for instance Grisvard (1985)). We also point out that condition (30) is automatically satisfied, since the considered problem is two-dimensional. On the other hand, Propositions 3.3 and 3.6 of Hetmaniuk (2007) show that the stability property of Definition 2 holds with $\alpha=0$.

Simple arguments show that the sesquilinear form $b_{k}$ introduced at (43) satisfies all the hypotheses of Section 2.2. Thus, as a direct application of our general analysis, i.e., Theorem 2, we can state the following results for the particular case of the Helmholtz equation.

Proposition 3. Assume that $\Gamma_{D}$ and $\Gamma_{A}$ are of class $C^{\gamma+1,1}$ and let $p \leq \gamma+1$. If $k^{p+1} h^{p}$ is small enough, then there exists a unique solution $u_{h, p} \in V_{h, p}$ of $b_{k}\left(u_{h, p}, v_{h, p}\right)=\left(f, v_{h, p}\right)$ for all $v_{h, p} \in V_{h, p}$ and we have

$$
\left\|\left|u-u_{h, p}\right|\right\| \mid \lesssim k h\|f\|_{0, \Omega} .
$$

In addition, if $f \in H^{p-1}(\Omega)$, then we have

$$
\left\|\left|u-u_{h, p}\right|\right\| \mid \lesssim k^{p} h^{p}\|f\|_{p-1, \Omega} .
$$

On the other hand, we can state the following result for the case of polygonal boundaries.

Proposition 4. Assume that $N=2$ and that $\Gamma_{D}$ and $\Gamma_{A}$ are polygonal and let $p \in \mathbb{N}$. If the meshes are refined near the corners of $\Gamma_{D}$ and $\Gamma_{A}$ according to (31)-(32), and if $k^{p+1} h^{p}$ is small enough, then the statements of Proposition 3 remain valid.

Remark 8. The case of a three-dimensional domain $\Omega$ when $\Gamma_{D}$ and/or $\Gamma_{A}$ have corners can be also treated using the results from Grisvard (1985); Dauge (1988), in particular condition (30) holds due to (Kozlov et al., 2001, §2.2 and 2.3).

Remark 9. Our analysis allows both $\Gamma_{D}$ and $\Gamma_{A}$ to exhibit corners. In contrast, previous works take into account corners in $\Gamma_{A}$ but only when $\Gamma_{D}$ is empty (Esterhazy 8 Melenk, 2012; Melenk 83 Sauter, 2011).

\section{Application to the time-harmonic elastodynamic System}

We consider time harmonic elastic wave propagation in an homogeneous (possibly) anisotropic medium $\Omega \subset \mathbb{R}^{3}$. We assume that the boundary of $\Omega$ is sufficiently smooth, namely of class $C^{\gamma+1,1}$. For the sake of simplicity we restrict ourselves to three-dimensional problems, but it is easy to check that the results presented below remain valid for two-dimensional problems.

The elasticity properties of the material are represented by a constant second-order tensor $C=\left(c_{n m p q}\right)_{n, m, p, q=1}^{3}$ satisfying the following symmetry and ellipticity properties

$$
c_{n m p q}=c_{m n p q}=c_{p q n m}, \quad 1 \leq n, m, p, q \leq 3,
$$

and

$$
\sum_{n, m, p, q=1}^{3} c_{n m p q} \varepsilon_{n m} \varepsilon_{p q} \gtrsim \sum_{n, m=1}^{3}\left|\varepsilon_{n m}\right|^{2},
$$

for all first-order symmetric tensors $\varepsilon=\left(\varepsilon_{n m}\right)_{n, m=1}^{3}$. 
For a given displacement $v: \Omega \rightarrow \mathbb{C}^{3}$, the strain and stress tensors are respectively defined by

$$
\varepsilon(u)=\frac{1}{2}\left(\nabla u+(\nabla u)^{\top}\right)
$$

and

$$
\sigma(u)=C \varepsilon(u) .
$$

The unknown $u: \Omega \rightarrow \mathbb{C}^{3}$ represents the displacement under the action of a load term $f: \Omega \rightarrow$ $\mathbb{C}^{3}$. $u$ and $f$ are related trough the elastodynamic system

$$
\left\{\begin{array}{rlll}
-k^{2} u-\nabla \cdot \sigma(u) & =f & & \text { in } \Omega, \\
\sigma(u) n-i k A u & =0 & & \text { on } \Gamma_{A}, \\
u & =0 & & \text { on } \Gamma_{D},
\end{array}\right.
$$

where $k$ is the (real) wavenumber, $A: \Gamma_{A} \rightarrow \mathcal{M}\left(\mathbb{R}^{3}\right)$ is a matrix-valued functions of class $C^{\gamma, 1}$ that is supposed to be symmetric and uniformly positive definite, i.e.

$$
Y^{\top} A(x) Y \gtrsim|Y|^{2}, \quad \forall Y \in \mathbb{R}^{3}, \quad \forall x \in \Gamma_{A},
$$

where the hidden constant does not depend on $x$.

Though our stability results and error estimates hold for arbitrary matrix-valued functions $A$ satisfying the previous assumptions, it is clear that the efficiency of the absorbing boundary condition heavely depends on $A$. As a result, the matrix $A$ must be carefully designed in applications. For the special case of tilted transverse isotropic media, we refer the reader to Barucq et al. (2014) and Boillot et al. (2015). For the simplest case of an isotropic medium, a possible definition of $A$ is given in Clayton \& Engquist (1977), Darbas et al. (2017) and Lysmer \& Kuhlemeyer (1969), corresponding to the so-called Lysmer-Kuhlemeyer absorbing boundary condition. But in both cases, the symmetry and uniform positive definiteveness of $A$ are respected. In addition, since in the above examples, $A$ is expressed in terms of normal and tangential vectors to the boundary, it is reasonable to assume that $A$ has the regularity $C^{\gamma, 1}$, since the boundary is $C^{\gamma+1,1}$.

4.1. Well-posedness results. We introduce the variational formulation of problem (44). It reads: find $u \in H_{\Gamma_{D}}^{1}(\Omega)^{3}$ such that

$$
b_{k}(u, v)=(f, v), \quad \forall v \in H_{\Gamma_{D}}^{1}(\Omega)^{3} .
$$

where $f \in L^{2}(\Omega)^{3}$ and

$$
b_{k}(u, v)=-k^{2}(u, v)-i k\langle A u, v\rangle_{\Gamma_{A}}+(C \varepsilon(u), \varepsilon(v)) .
$$

We easily see that $b_{k}$ satisfies Assumption 1. Indeed, continuity (25) follows from a trace theorem, and Gårding inequality (26) is a consequence of Korn's inequality.

We now check whether the shift property from Definition 1 is valid for the corresponding system (5), that here takes the form

$$
\left\{\begin{aligned}
-\nabla \cdot \sigma(\tilde{u}) & =\tilde{f} & & \text { in } \Omega \\
\sigma(\tilde{u}) n & =\tilde{g} & & \text { on } \Gamma_{A} \\
\tilde{u} & =0 & & \text { on } \Gamma_{D} .
\end{aligned}\right.
$$

Since this system corresponds to (44) with $k=0$, its variational formulation is clearly (45) with $k=0$ and the right-hand-side

$$
\langle\psi, v\rangle_{-1,1}=(\tilde{f}, v)+(\tilde{g}, v)_{\Gamma_{A}}, \forall v \in H_{\Gamma_{D}}^{1}(\Omega)^{3} .
$$

As we have just checked that $b_{0}$ satisfies Gårding inequality $(26)$, it will be coercive on $H_{\Gamma_{D}}^{1}(\Omega)^{3}$ if $\Gamma_{D}$ is non-empty since Poincaré's inequality guarantees that

$$
|w|_{1, \Omega}^{2} \gtrsim\|w\|_{1, \Omega}^{2}, \forall w \in H_{\Gamma_{D}}^{1}(\Omega) .
$$

In this case, problem (46) is well-posed in $H_{\Gamma_{D}}^{1}(\Omega)^{3}$. 
The situation is more delicate if $\Gamma_{D}$ is empty, because the well-posedness of (46) is subject to some orthogonality conditions on $\tilde{f}, \tilde{g}$. Hence in this case, we perform a change of parameter, namely we set $\kappa=k-i$. Then we transform problem (44) into

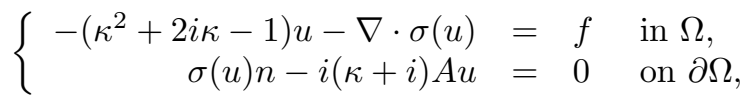

that enters into our abstract setting with $\delta=1$,

$$
\mathcal{L}_{2} u=u-\nabla \cdot \sigma(u), \mathcal{L}_{1} u=2 u, \mathcal{L}_{0}=u,
$$

and

$$
\mathcal{B}_{A}=\sigma(u) n+A u \text {. }
$$

In that situation, the corresponding system (5) is

$$
\left\{\begin{aligned}
\tilde{u}-\nabla \cdot \sigma(\tilde{u}) & =\tilde{f} & & \text { in } \Omega \\
\sigma(\tilde{u}) n+A \tilde{u} & =\tilde{g} & & \text { on } \partial \Omega
\end{aligned}\right.
$$

whose variational formulation is given by

$$
b_{0}(\tilde{u}, v)=(\tilde{f}, v)+(\tilde{g}, v)_{\Gamma_{A}}, \forall v \in H^{1}(\Omega)^{3},
$$

with

$$
b_{0}(u, v)=(u, v)+\langle A u, v\rangle_{\partial \Omega}+(C \varepsilon(u), \varepsilon(v)) .
$$

Since

$$
b_{0}(u, u)=\|u\|_{0, \Omega}^{2}+(C \varepsilon(u), \varepsilon(u))+\langle A u, u\rangle_{\partial \Omega}
$$

by our assumption on $A$, we clearly have

$$
b_{0}(u, u) \geq\|u\|_{0, \Omega}^{2}+(C \varepsilon(u), \varepsilon(u))
$$

and by Korn's inequality we conclude that $b_{0}$ is coercive on $H^{1}(\Omega)^{3}$. Consequently, problem (48) is well-posed in $H^{1}(\Omega)^{3}$.

Once the well-posedness in $H^{1}(\Omega)^{3}$ is established, the shift property for both problems (46) and (48) holds owing to standard theory of elliptic systems (the reader will find more details in classical text book such as Lions \& Magenes (1968) and Nečas (1967), as well as $\S 4.4$ of Costabel et al. (2010)) if the boundary of $\Omega$ is of class $C^{\gamma+1,1}$ and if $\bar{\Gamma}_{A} \cap \bar{\Gamma}_{D}=\emptyset$. The shift property is also valid for less regular boundaries or if $\bar{\Gamma}_{A} \cap \bar{\Gamma}_{D}$ is non-empty, see for instance Maz'ya \& Rossmann (2010).

Remark 10. If the elastic tensor corresponds to the Lamé operator, then the case when $\Gamma_{D}$ and/or $\Gamma_{A}$ have corners can be also treated using the results from Grisvard (1986); Dauge (1988), in particular condition (30) holds due to (Kozlov et al., 2001, §3.4 and 4.3).

4.2. Stability property. The elasticity system also satisfies the $k$-stability property of Definition 2 , as we show in the next proposition. Our proof is a simple consequence of a result obtained in Lagnese (1983) for the time-dependent elastodynamic system combined with the next result of functional analysis (see Huang, 1985; Prüss, 1984).

Lemma 4. $A C_{0}$ semigroup $\left(e^{t \mathcal{L}}\right)_{t \geq 0}$ of contractions on a Hilbert space $H$ is exponentially stable, i.e., satisfies

$$
\left\|e^{t \mathcal{L}} U_{0}\right\| \leq C e^{-\omega t}\left\|U_{0}\right\|_{H}, \quad \forall U_{0} \in H, \quad \forall t \geq 0
$$

for some positive constants $C$ and $\omega$ if and only if

$$
\rho(\mathcal{L}) \supset\{i \beta \mid \beta \in \mathbb{R}\} \equiv i \mathbb{R},
$$

and

$$
\sup _{\beta \in \mathbb{R}}\left\|(i \beta \mathbb{I}-\mathcal{L})^{-1}\right\|<\infty,
$$

where $\rho(\mathcal{L})$ denotes the resolvent set of the operator $\mathcal{L}$. 
Proposition 5. Assume that there exists $x_{0} \in \mathbb{R}^{3}$ such that

$$
\begin{gathered}
\left(x-x_{0}\right) \cdot n(x) \leq 0, \forall x \in \Gamma_{D}, \\
\left(x-x_{0}\right) \cdot n(x)>0, \forall x \in \bar{\Gamma}_{A} .
\end{gathered}
$$

Let $u \in H^{1}(\Omega)^{3}$ be a solution of (44) with a right-hand-side $f \in L^{2}(\Omega)^{3}$. Then $u \in H^{2}(\Omega)^{3}$ and

$$
k\|u\|_{0, \Omega}+|u|_{1, \Omega}+k^{-1}|u|_{2, \Omega} \lesssim\|f\|_{0, \Omega},
$$

where the hidden constant depends on the domain $\Omega$, the elastic tensor $C$ and the impedance matrix $A$, but is independent of $k$.

Remark 11. Assumption (51) states that $\Gamma_{D}$ is the boundary of a Dirichlet obstacle that is starshaped with respect to $x_{0}$. Similarly, Assumption (52) states that the domain enclosed by $\Gamma_{A}$ is star-shaped with respect to $x_{0}$. Since in applications, $\Gamma_{A}$ is usually designed as a sphere or a brick centered around the obstacle, in practice Assumption (52) is always satisfied. We can thus think of the problem considered in Proposition 5 as the general case of scattering by a star-shaped Dirichlet obstacle.

Remark 12. Other recent works focus on frequency-explicit stability bounds for Problem (44). Specifically, we can mention Theorem 2.3 of Brown 83 Gallistl (2016), where the authors derive a bound for general Lipschitz domains. Though this bound applies to a wider class of problems, it is less sharp when restricted to the assumptions of Proposition 5. We can also mention Cummings $\&$ Feng (2006), where the authors makes the same hypothesis than here, but obtain a bound that is less sharp.

Proof. Under our assumptions, the combination of Theorem 1.1 of Lagnese (1983) (valid for a diagonal matrix $A$ ) with Theorem 5.3 of Nicaise (2003) (that allows to pass from the case $A=I d$ to the general case of a uniformly positive definite matrix $A$, see Nicaise $(2003, \S 6.5)$ for more explanations) (see also Theorem 1.3 of Alabau \& Komornik (1998) and section 6.3 of Nicaise (2003)) implies that the time-dependent elastodynamic system

$$
\left\{\begin{array}{rlrl}
u_{t t}-\nabla \cdot \sigma(u) & =0 & & \text { in } \Omega \times(0, \infty), \\
\sigma(u) n-A u_{t} & =0 & & \text { on } \Gamma_{A} \times(0, \infty), \\
u & =0 & & \text { on } \Gamma_{D} \times(0, \infty), \\
u(\cdot, t=0) & =u_{0}, u_{t}(\cdot, t=0)=u_{1}, &
\end{array}\right.
$$

is exponential stable, in other words, its energy

$$
\frac{1}{2}\left(\int_{\Omega}\left(\left|u_{t}(\cdot, t)\right|^{2}+\sigma(u(\cdot, t)): \varepsilon(u(\cdot, t))\right) d x\right)
$$

decays exponentially (as $t \rightarrow \infty$ ). In order to make use of Lemma 4, we use the standard reduction of order. Setting $U=\left(u, u_{t}\right)^{\top}$, this system is equivalent to the first order one

$$
U_{t}=\mathcal{A} U \text { in } \mathcal{H}, t>0, U(t=0)=\left(u_{0}, u_{1}\right)^{\top},
$$

where the Hilbert space $\mathcal{H}$ and the operator $\mathcal{A}$ are defined as follows. Let us set

$$
\mathcal{H}=H_{\Gamma_{D}}^{1}(\Omega)^{3} \times L^{2}(\Omega)^{3} .
$$

If $\Gamma_{D}$ is non-empty, $\mathcal{H}$ is equipped with the norm

$$
\left\|(u, v)^{\top}\right\|_{\mathcal{H}}^{2}:=\int_{\Omega}\left(|v|^{2}+\sigma(u): \varepsilon(\bar{u})\right) d x, \quad \forall(u, v)^{\top} \in \mathcal{H} .
$$

On the contrary $\mathcal{H}$ is equipped with the norm

$$
\left\|(u, v)^{\top}\right\|^{2}:=\left\|(u, v)^{\top}\right\|_{\mathcal{H}}^{2}+\int_{\partial \Omega} A u \cdot \bar{u} d \sigma(x), \quad \forall(u, v)^{\top} \in \mathcal{H},
$$

when $\Gamma_{D}$ is empty.

In both cases, the norm property is based on Korn's inequality (see Theorem 3.3 of Duvaut \& Lions (1972) or Theorem 2.1 of Ciarlet (2010)). Now we define

$$
D(\mathcal{A})=\left\{(u, v)^{\top} \in \mathcal{H}: v \in H_{\Gamma_{D}}^{1}(\Omega)^{3}, u \in H^{2}(\Omega)^{3} \text { satisfying } \sigma(u) n+A v=0 \text { on } \Gamma_{A}\right\},
$$


and set

$$
\mathcal{A}(u, v)^{\top}=(v, \nabla \cdot \sigma(u)), \quad \forall(u, v)^{\top} \in D(\mathcal{A}) .
$$

It is well know that $\mathcal{A}$ generates a $C_{0}$ semigroup $\left(e^{t \mathcal{A}}\right)_{t \geq 0}$ of contractions on $\mathcal{H}$, see for instance p. 972 of Lagnese (1983) (as conditions (51)-(52) imply that $\bar{\Gamma}_{A} \cap \bar{\Gamma}_{D}=\emptyset$ ). Hence by the exponential decay of the energy and Lemma 4 , when $\Gamma_{D}$ is non empty we deduce that the resolvent of $\mathcal{A}$ is uniformly bounded in the imaginary axis. In other words for all $\left(g_{1}, f_{1}\right)^{\top} \in \mathcal{H}$ and all $\xi \in \mathbb{R}$, there exists a unique solution $(u, v)^{\top} \in D(\mathcal{A})$ of

$$
(i \xi \mathbb{I}-\mathcal{A})(u, v)^{\top}=\left(g_{1}, f_{1}\right)^{\top},
$$

with

$$
\left\|(u, v)^{\top}\right\|_{\mathcal{H}} \lesssim\left\|\left(g_{1}, f_{1}\right)^{\top}\right\|_{\mathcal{H}} .
$$

This equivalently means that $(u, v)^{\top} \in D(\mathcal{A})$ satisfies

$$
\begin{array}{r}
i \xi u-v=g_{1} \text { in } \Omega, \\
i \xi v-\nabla \cdot \sigma(u)=f_{1} \text { in } \Omega .
\end{array}
$$

The first identity means that

$$
v=i \xi u-g_{1} \text { in } \Omega,
$$

and eliminating $v$ in the second equation we find that $u$ is solution to

$$
-\xi^{2} u-\nabla \cdot \sigma(u)=f_{1}+i \xi g_{1} \text { in } \Omega .
$$

Reminding the boundary conditions satisfied by $(u, v)^{\top} \in D(\mathcal{A})$, namely $u=0$ on $\Gamma_{D}$ and

$$
\sigma(u) n+A v=0 \text { on } \Gamma_{A},
$$

and using (56) we find that

$$
\sigma(u) n+i \xi A u=A g_{1} \text { on } \Gamma_{A} .
$$

Taking $g_{1}=0$ and $f_{1}=f$, we see that $u$ is solution of (44) if we chose $\xi=-k$. Coming back to (55), using Korn's inequality and again using (56) with $g_{1}=0$, one deduces the estimate

$$
\|u\|_{1, \Omega}+k\|u\|_{0, \Omega} \lesssim\|f\|_{0, \Omega}
$$

As $u$ can be seen as the solution of

$$
\left\{\begin{aligned}
-\nabla \cdot \sigma(u) & =f+k^{2} u & & \text { in } \Omega \\
\sigma(u) n & =i k A u & & \text { on } \Gamma_{A} \\
u & =0 & & \text { on } \Gamma_{D}
\end{aligned}\right.
$$

by elliptic regularity we find that

$$
\|u\|_{2, \Omega} \lesssim\|f\|_{0, \Omega}+k^{2}\|u\|_{0, \Omega}+k\|u\|_{1 / 2, \Gamma_{A}} .
$$

By a trace theorem and estimate (60), we conclude that

$$
\|u\|_{2, \Omega} \lesssim k\|f\|_{0, \Omega} \text {. }
$$

This estimate and (60) lead to (53).

The situation is more delicate in the case $\Gamma_{D}=\emptyset$. Indeed, the quantity $\|\cdot\|_{\mathcal{H}}$ is no more a norm in $\mathcal{H}$ (hence the exponential decay of $\|\left(u(\cdot, t), v(\cdot, t) \|_{\mathcal{H}}\right.$ does not allow to apply directly Lemma 4). Indeed in such a case

$$
\left\|(u, v)^{\top}\right\|_{\mathcal{H}}=0
$$

if and only if $v=0$ and $u \in \mathcal{R}$, where $\mathcal{R}$ is the set of rigid displacement given by

$$
\mathcal{R}=\left\{\mathbf{a} \times x+\mathbf{b}: \mathbf{a}, \mathbf{b} \in \mathbb{C}^{3}\right\},
$$

and is of dimension 6 .

Hence we introduce the subspace

$$
\mathcal{H}_{0}=\left\{(u, v)^{\top} \in \mathcal{H} ; \int_{\Omega} v \cdot \bar{r} d x+\int_{\Gamma} A u \cdot \bar{r} d \sigma(x)=0, \forall r \in \mathcal{R}\right\} .
$$


The introduction of this subspace has two main features. First

$$
(\mathcal{R} \times\{0\}) \cap \mathcal{H}_{0}=\{0\},
$$

and hence the semi-norm $\|\cdot\|_{\mathcal{H}}$ is a norm on $\mathcal{H}_{0}$. Indeed if $(u, v)^{\top} \in(\mathcal{R} \times\{0\}) \cap \mathcal{H}_{0}$, then $v=0$ and $u \in H^{1}(\Omega)^{3} \cap \mathcal{R}$ satisfies

$$
\int_{\Gamma} A u \cdot \bar{r} d \sigma(x)=0, \quad \forall r \in \mathcal{R}
$$

In particular, it yields

$$
\int_{\Gamma} A u \cdot \bar{u} d \sigma(x)=0
$$

and consequently $u=0$, since the quantity

$$
\left(\int_{\Gamma} A u \cdot \bar{u} d \sigma(x)\right)^{1 / 2}
$$

is a norm on $\mathcal{R}$. Second, we see that

$$
\mathcal{A}(u, v)^{\top} \in \mathcal{H}_{0}, \quad \forall(u, v)^{\top} \in D(\mathcal{A}) .
$$

Indeed for $(u, v)^{\top} \in D(\mathcal{A})$, we have $\mathcal{A}(u, v)^{\top}=(v, \nabla \cdot \sigma(u))$, hence (61) holds if and only if

$$
\int_{\Omega} \nabla \cdot \sigma(u) \cdot \bar{r} d x+\int_{\Gamma} A v \cdot \bar{r} d \sigma(x)=0 .
$$

But according to Green's formula, we have

$$
\int_{\Omega} \nabla \cdot \sigma(u) \cdot \bar{r} d x=\int_{\Gamma} \sigma(u) n \cdot \bar{r} d \sigma(x)
$$

and using the boundary condition (58), we find that

$$
\int_{\Omega} \nabla \cdot \sigma(u) \cdot \bar{r} d x=-\int_{\Gamma} A v \cdot \bar{r} d \sigma(x),
$$

which proves (62).

This justifies the introduction of the operator $\mathcal{A}_{0}$ that is the restriction of $\mathcal{A}$ to $\mathcal{H}_{0}$. Trivially this restriction generates a $C_{0}$ semigroup of contractions on $\mathcal{H}_{0}$. Hence by the exponential decay of the energy and Lemma 4 (applied to $\mathcal{A}_{0}$ in $\mathcal{H}_{0}$ ), we deduce that the resolvent of $\mathcal{A}_{0}$ is uniformly bounded on the imaginary axis, in other words, for any $\left(g_{1}, f_{1}\right)^{\top} \in \mathcal{H}_{0}$ and all $\xi \in \mathbb{R}$, there exists a unique solution $(u, v)^{\top} \in D\left(\mathcal{A}_{0}\right)$ of (54) with the estimate (55). As before, we deduce that $v$ is given by (56) and that $u$ is solution of (57) and (59). The problem is that the pair $\left(g_{1}, f_{1}\right)$ fulfils the condition

$$
\int_{\Omega} f_{1} \cdot \bar{r} d x+\int_{\Gamma} A g_{1} \cdot \bar{r} d \sigma(x)=0, \quad \forall r \in \mathcal{R},
$$

and that we can no more choose $g_{1}=0$, since we want to take arbitrary right-hand side $f$ in (44). The solution is to take

$$
g_{1}=\varphi \sum_{j=1}^{6} \alpha_{j} r_{j}, f_{1}=f-i \xi g_{1},
$$

with $\alpha_{j} \in \mathbb{C}$ determined below and a fixed function $\varphi \in \mathcal{D}(\Omega)$ such that $\varphi=1$ in an open non empty subset $O$ of $\Omega$ and $\left\{r_{j}\right\}_{j=1}^{6}$ is a basis of $\mathcal{R}$ such that

$$
\int_{\Omega} \varphi r_{j} \cdot \bar{r}_{k} d x=\delta_{j, k}, \quad \forall j, k=1, \cdots, 6 .
$$

This is possible because

$$
\int_{\Omega} \varphi r \cdot \bar{s} d x
$$

is an inner product on $\mathcal{R}$. 
With these choices, if $\xi \neq 0$, we see that condition (63) holds if and only if ( since $g_{1}=0$ on $\Gamma$ )

$$
\int_{\Omega}\left(f-i \xi g_{1}\right) \bar{r}_{j} d x=0, \forall j=1, \cdots, 6,
$$

or equivalently using (65),

$$
\alpha_{j}=-\frac{1}{i \xi} \int_{\Omega} f \bar{r}_{j} d x, \forall j=1, \cdots, 6 .
$$

By choosing $\xi=-k$, we then deduce that $u$ is solution of (44). It then remains to prove (60). But reminding the estimate (55) and the choice (64), we have

$$
\left\|(u, v)^{\top}\right\|_{\mathcal{H}} \lesssim(1+k) \sum_{j=1}^{6}\left|\alpha_{j}\right|+\|f\|_{0, \Omega} .
$$

But (66) and Cauchy-Schwarz's inequality yield

$$
\left|\alpha_{j}\right| \lesssim \frac{1}{k}\|f\|_{0, \Omega}, \forall j=1, \cdots, 6
$$

and therefore the previous estimate becomes

$$
\left\|(u, v)^{\top}\right\|_{\mathcal{H}} \lesssim\|f\|_{0, \Omega}
$$

This yields the estimates

$$
\begin{array}{r}
\left(\int_{\Omega} \sigma(u): \varepsilon(u) d x\right)^{\frac{1}{2}} \lesssim\|f\|_{0, \Omega} \\
\|v\|_{0, \Omega} \leq C_{3}\|f\|_{0, \Omega}
\end{array}
$$

and to catch the $L^{2}$-norm of $u$, we exploit the second estimate. Indeed (56) yields

$$
\|u\|_{0, \Omega} \leq \frac{1}{k}\left(\|v\|_{0, \Omega}+\left\|g_{1}\right\|_{0, \Omega}\right)
$$

hence by (69), (64) and (67), one obtains

$$
\|u\|_{0, \Omega} \lesssim \frac{1}{k}\|f\|_{0, \Omega} .
$$

This estimate and (68) lead to (60). The estimate of the $H^{2}$-norm of $u$ being the same than before, the proof is complete.

Remark 13. If $\Gamma_{D}$ is non empty, the previous result directly furnishes the stability property for system (44). On the contrary if $\Gamma_{D}$ is empty, it indirectly furnishes the stability property for system (47), since this last system is equivalent to (44).

4.3. Finite element discretization. In order to use Theorem 2 for conforming finite element discretizations of the elastodynamic system described in Subsection 2.2, it remains to check that $\mathcal{P}_{k}^{\star}$ satisfies the assumptions from Definitions 1 and 2 .

For that purpose, we characterize the boundary value problem associated with $\mathcal{P}_{k}^{\star}$. Consider an arbitrary $v \in H_{\Gamma_{D}}^{1}(\Omega)^{3}$ and let $g=\mathcal{P}_{k} v \in\left(H_{\Gamma_{D}}^{1}(\Omega)^{3}\right)^{\prime}$. Then, for all $\phi \in H_{\Gamma_{D}}^{1}(\Omega)^{3}$, we have

$$
\left\langle\phi, \mathcal{P}_{k}^{\star} \bar{v}\right\rangle_{1,-1}=b_{k}(\phi, \bar{v})=b_{k}(v, \bar{\phi})=\left\langle\mathcal{P}_{k} v, \bar{\phi}\right\rangle_{-1,1}=\langle g, \bar{\phi}\rangle_{-1,1}=\langle\phi, \bar{g}\rangle_{1,-1},
$$

so that $\mathcal{P}_{k} \bar{v}=\bar{g}$. Hence, since $\mathcal{P}_{k}$ satisfies the assumptions from Definitions 1 and 2 , so does $\mathcal{P}_{k}^{\star}$.

As a result, Theorem 2 holds for conforming finite element discretizations of the elastodynamic system. 
4.4. Numerical experiments. We consider a toy experiment in the unit square $\Omega=(0,1)^{2}$ to illustrate our results. For additional experiments, we refer to Darbas et al. (2017), where the performance of the Lysmer-Kuhlemeyer absorbing boundary condition is compared with a higher order absorbing boundary condition (that is not covered by our framework). We consider an isotropic elastic medium with Lamé parameters $\lambda=\mu=1$ and density $\rho=1$. We set very simple boundary conditions corresponding to the case $A=I d$ and $\Gamma_{D}=\emptyset$. We consider an analytic solution that is the superposition of a $\mathrm{P}$ wave and a $\mathrm{S}$ wave travelling in different directions:

$$
\begin{aligned}
u(x) & =\left(\cos \left(\theta_{p}\right), \sin \left(\theta_{p}\right)\right) \frac{1}{k} \exp \left(\frac{i k}{\sqrt{\lambda+2 \mu}}\left(\cos \left(\theta_{p}\right) x_{1}+\sin \left(\theta_{p}\right) x_{2}\right)\right) \\
& +\left(-\sin \left(\theta_{s}\right), \cos \left(\theta_{s}\right)\right) \frac{1}{k} \exp \left(\frac{i k}{\sqrt{\mu}}\left(\cos \left(\theta_{s}\right) x_{1}+\sin \left(\theta_{s}\right) x_{2}\right)\right),
\end{aligned}
$$

with $\theta_{p}=\pi / 3$ and $\theta_{s}=-\pi / 3$.

Since these plane waves satisfy the homogeneous equation inside $\Omega$, we use inhomongeous boundary condition to impose them as the solution. Hence, the problem read:

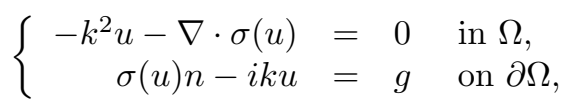

where

and $g=\sigma(u) n-i k u$.

$$
\sigma(u)=(\nabla \cdot u) I+\varepsilon(u)
$$

Remark 14. Even if the boundary of $\Omega$ is not smooth, the shift property from Definition 1 holds for all $\gamma \geq 0$. Indeed in such a situation, according to Theorem 4.1.1 from Grisvard (1986), the weak solution $\tilde{u} \in H^{1}(\Omega)^{2}$ of

$$
\left\{\begin{aligned}
-\nabla \cdot \sigma(\tilde{u}) & =\tilde{f} \quad \text { in } \Omega, \\
\sigma(\tilde{u}) n & =\tilde{g}
\end{aligned}\right.
$$

with $\tilde{f} \in H^{l}(\Omega)^{2}, \tilde{g} \in H^{l+1 / 2}(\partial \Omega)^{2}$ belongs to $A^{l+2}=H^{l+2}(\Omega)^{2} \oplus$ span $S^{\lambda}$, where $S^{\lambda}$ are the singular functions associated with system (71) (see (6)-(7)) and $\lambda \in \mathbb{C}$ is a root of the transcendental equation

$$
\sin ^{2}\left(\frac{\lambda \pi}{2}\right)=\lambda^{2}
$$

Note that according to Theorems 4.2.1 and 4.2.2 of Kozlov et al. (2001), the strip $\operatorname{Re} \lambda \in[0,2]$ is free of such a root and consequently the weak solution belongs to $H^{l+2}(\Omega)^{2}$ for $l=0$ or 1 . On the contrary for $l \geq 2$, Theorem 4.2.3 of Kozlov et al. (2001) states that the strip $\operatorname{Re} \lambda \in(0, l+1)$ contains such a root and therefore the solution of (71) does not belong to $H^{l+2}(\Omega)^{2}$ in general. Let us also remark that the construction of the singular spaces $\mathcal{T}_{c, j}^{\lambda}$ directly follows from a logarithmopolynomial resolution, see (Nicaise, 1993, §4.3, 4.4), since the involved operators have constant coefficients (see (11) and (12)).

We need to quantify the best approximation of the scheme. To this end, we will also solve the auxiliary problem to find $w_{h, p} \in V_{h, p}$

$$
k^{2}\left(w_{h, p}, \phi_{h, p}\right)+\left(\nabla w_{h, p}, \nabla \phi_{h, p}\right)=k^{2}\left(u, \phi_{h, p}\right)+\left(\nabla u, \nabla \phi_{h, p}\right)
$$

for all $\phi_{h, p} \in V_{h, p}$. One observes that this $w_{h, p}$ satisfies

$$
\left\|\left|u-w_{h, p}\right|\right\|=\min _{\phi_{h, p} \in V_{h, p}}\left\|u-\phi_{h, p}\right\| \mid .
$$

The main result of our theoretical analysis states that if $k^{p+1} h^{p} \lesssim 1$ and for $p \geq 2$, if the meshes are appropriately refined near the corners, then

$$
||\left|u-u_{h, p}\right||| \lesssim||\left|u-w_{h, p}\right||| .
$$

With this experiment, we illustrate that this bound is sharp. To do so, we compute $u_{h, p}$ and $w_{h, p}$ for different $k$ and different quasi-uniform meshes of meshsize $h$. More precisely, we first fix a value of $k$, and then compute the finite element solution and projection for decreasing values of 
$h$, as depicted on Figure 1 and 2. These figures illustrate that as expected, the projection $w_{h, p}$ converges as $h$ and $h^{4}$ to the solution $u$ for $\mathbb{P}_{1}$ and $\mathbb{P}_{4}$ elements respectively. In addition, the finite element solution $u_{h, p}$ is almost as accurate as the projection $w_{h, p}$ for small values of $h$. However, a gap is present for largest values of $h$. One also sees that this gap increases as $k$ increases.

We proceed as follow to determine if (72) is sharp. For each $k$, we denote by $h^{\star}(k)$ the greatest value $h_{0}$ such that

$$
\left\|\left|u-u_{h, p}\right|\right\| \leq 2\left|\left\|u-w_{h, p}\right\|\right|, \quad \forall h \leq h_{0} .
$$

The value of $h^{\star}(k)$ for a given $k$ is obtained by inspecting the corresponding convergence curve (see Figures 1 and 2). Condition (73) states that the finite element solution must be quasi optimal in the ||$|\cdot|||$-norm, uniformly in $k$ (with the arbitrary constant 2).

The graph of $h^{\star}(k)$ is represented on Figure 3 for $\mathbb{P}_{1}$ and $\mathbb{P}_{4}$ elements. We observe that in both cases $h^{\star}(k) \sim k^{-1-1 / p}$, which is in accordance with (72). Indeed, it means that quasi-optimality in the sense of (73) is achieved under the condition that $h \leq h^{\star}(k) \sim k^{-1-1 / p}$, which is equivalent to $k^{p+1} h^{p} \leq k^{p+1}\left[h^{\star}(k)\right]^{p} \lesssim 1$. We thus conclude that the stability condition presented in Theorem 2 is sharp even for $p=4$ with quasi-uniform meshes.

Note that our experiments indicate that the first statement from Theorem 2 may remain valid for values of $p$ larger than the theoretical one, that is here equal to 2 .

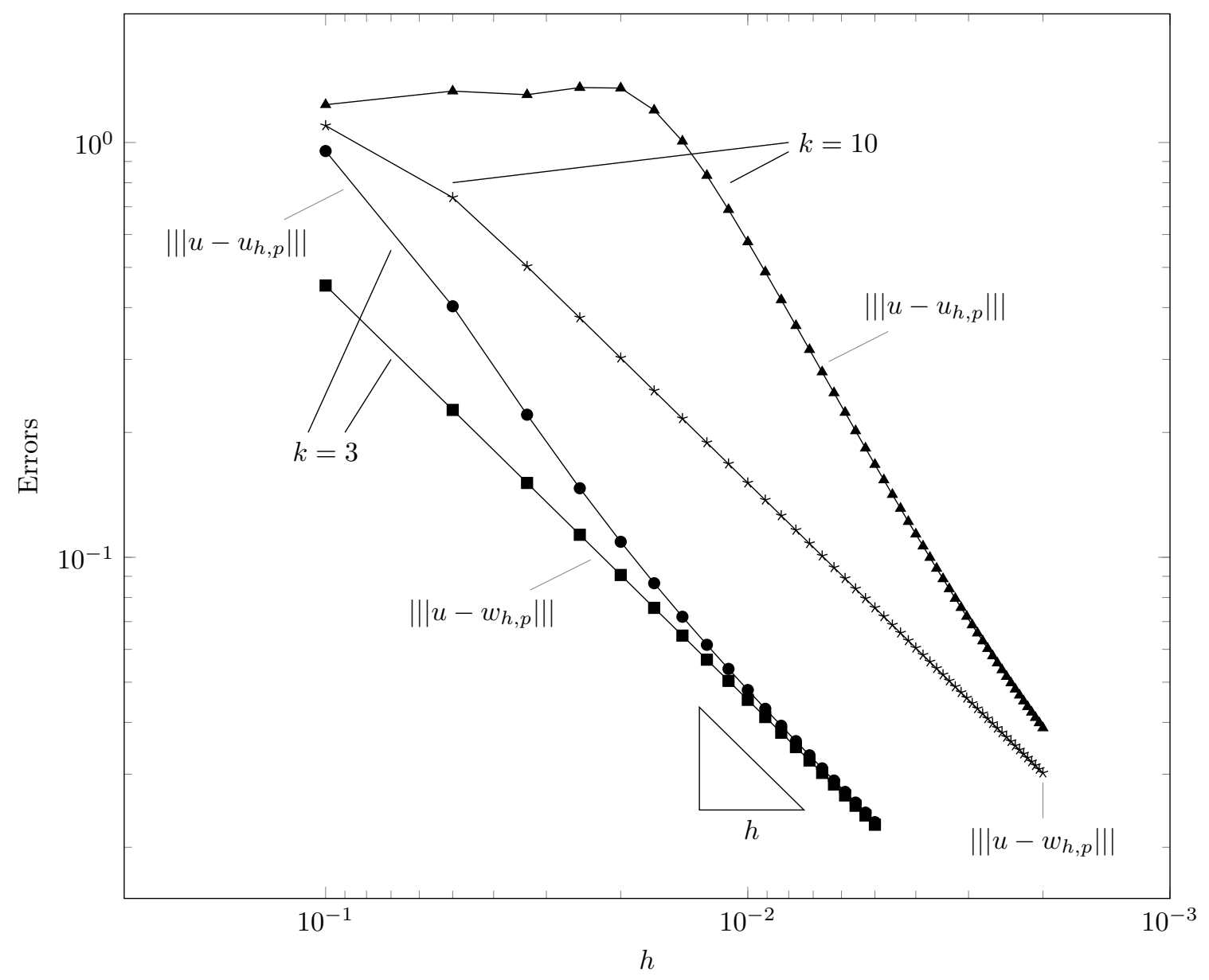

Figure 1. Convergence curves for $\mathbb{P}_{1}$ elements 


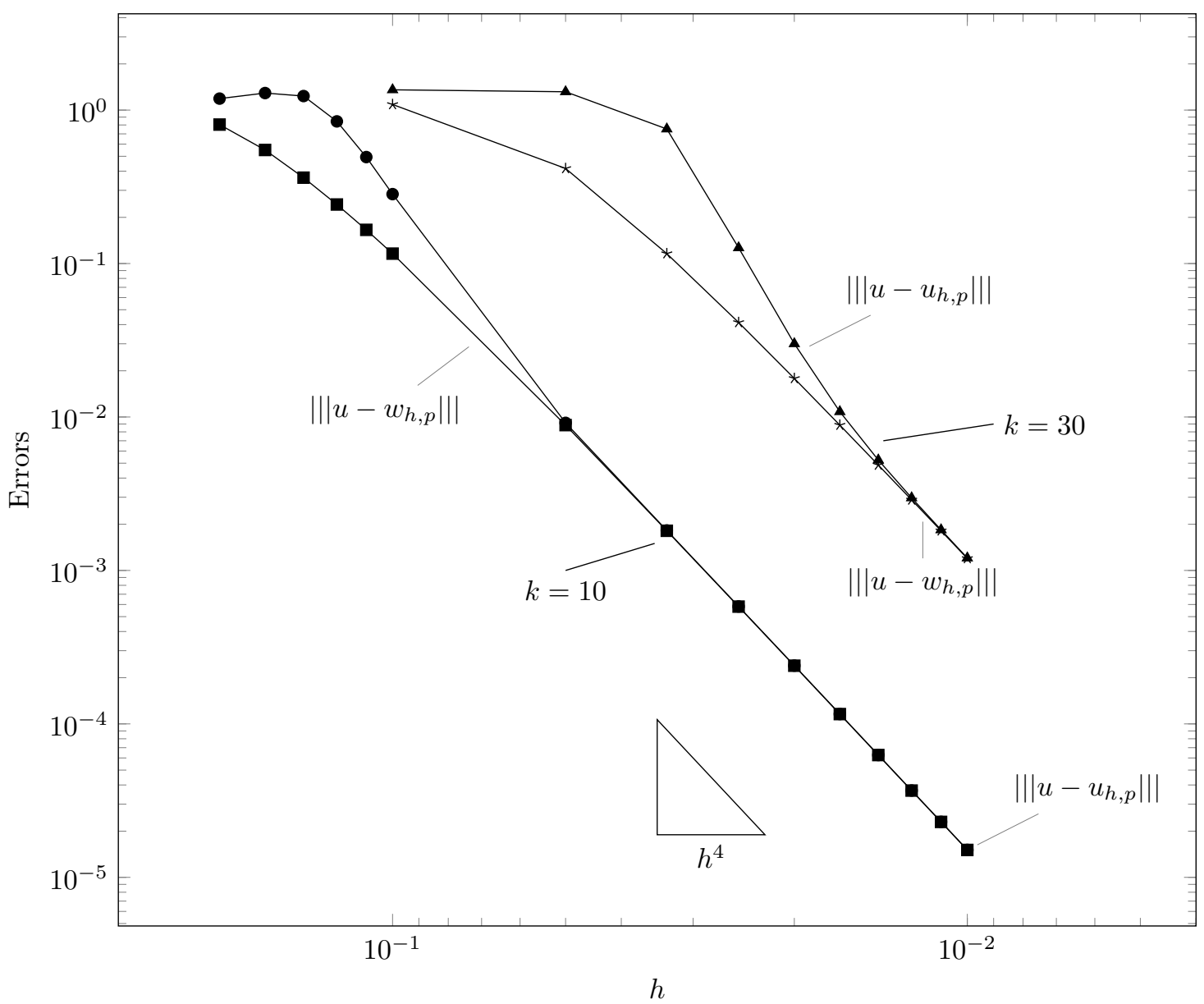

Figure 2. Convergence curves for $\mathbb{P}_{4}$ elements
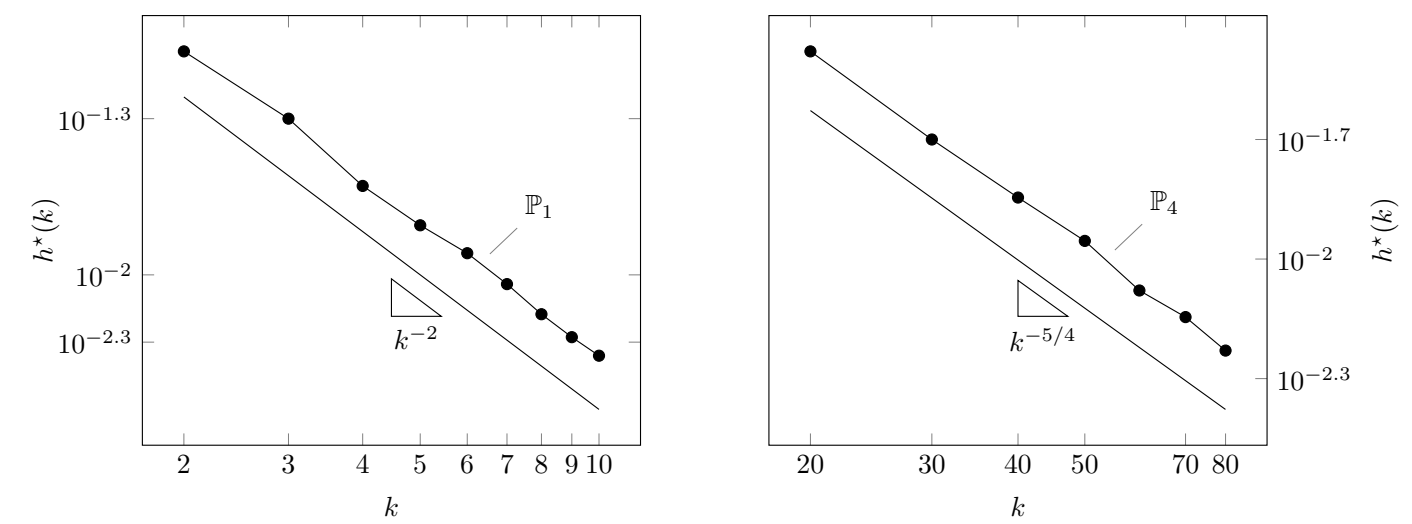

Figure 3. Elastic wave equation: asymptotic ranges $h^{\star}(k)$ for $\mathbb{P}_{1}$ (left) and $\mathbb{P}_{4}$ (right) elements

\section{Application to the convected Helmholtz equation}

We now consider the convected Helmholtz equation in a fluid with uniform horizontal motion (see, e.g., Bécache et al., 2004). The equation reads:

$$
\left\{\begin{array}{rlr}
-k^{2} u-2 i k M \frac{\partial u}{\partial \mathbf{x}_{1}}-\left(1-M^{2}\right) \frac{\partial^{2} u}{\partial \mathbf{x}_{1}^{2}}-\frac{\partial^{2} u}{\partial \mathbf{x}_{2}} & =f & \text { in } \Omega \\
\left(1-M^{2}\right) \frac{\partial u}{\partial \mathbf{x}_{1}} n_{1}+\frac{\partial u}{\partial \mathbf{x}_{2}} n_{2}-i k\left(1-M n_{1}\right) u & =0 & \text { on } \Gamma_{A} \\
u & =0 & \text { on } \Gamma_{D}
\end{array}\right.
$$


where $M \in(-1,1)$ is the Mach number, $\Omega=(0,1)^{2}$ is the unit square and $\Gamma_{D}=(0,1) \times\{0\}$ denotes its lower edge. The absorbing boundary condition on $\Gamma_{A}=\partial \Omega \backslash \overline{\Gamma_{D}}$ is designed to perfectly filter incoming "plane wave" travelling normally to the boundary.

Problem (74) fits in our general framework with $S=1$,

and

$$
\mathcal{L}_{0} u=u, \quad \mathcal{L}_{1} u=2 M \frac{\partial u}{\partial \mathbf{x}_{1}}, \quad \mathcal{L}_{2} u=-\left(1-M^{2}\right) \frac{\partial^{2} u}{\partial \mathbf{x}_{1}}-\frac{\partial^{2} u}{\partial \mathbf{x}_{2}},
$$

$$
\mathcal{B}_{D} u=u, \quad \mathcal{B}_{A} u=\left(1-M^{2}\right) \frac{\partial u}{\partial \mathbf{x}_{1}} n_{1}+\frac{\partial u}{\partial \mathbf{x}_{2}} n_{2}, \quad A u=\left(1-M n_{1}\right) u
$$

Since $|M|<1$, it is clear that the triplet $\left(\mathcal{L}_{2}, \mathcal{B}_{A}, \mathcal{B}_{D}\right)$ satisfies the shift property (introduced in Definition 1 ) at the order 3 with $\mathcal{S}_{c, j}^{\lambda}=\emptyset$. In addition, it is easily seen that $\mathcal{L}_{0}$ and $\mathcal{L}_{1}$ are consistent with (10). Thus, we only need to verify that the assumptions from Definition 2 hold. For that purpose, we perform a change of unknown in order to get back to a standard Helmholtz problem.

Lemma 5. Assume that $u \in H^{1}(\Omega)$ solves (74). Then the function $\tilde{u} \in H^{1}(\Omega)$ defined as

$$
\tilde{u}\left(\mathbf{x}_{1}, \mathbf{x}_{2}\right)=u\left(\mathbf{x}_{1}, \frac{\mathbf{x}_{2}}{\beta}\right) e^{-\rho \mathbf{x}_{1}}
$$

is solution to

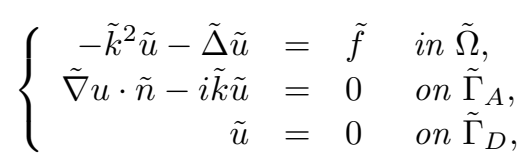

where

and

$$
\begin{gathered}
\tilde{\Omega}=(0,1) \times\left(0, \frac{1}{\beta}\right), \quad \tilde{\Gamma}_{D}=(0,1) \times\{0\}, \quad \tilde{\Gamma}_{A}=\tilde{\Omega} \backslash \overline{\tilde{\Gamma}_{D}} \\
\tilde{f}\left(\mathbf{x}_{1}, \mathbf{x}_{2}\right)=\frac{1}{1-M^{2}} f\left(\mathbf{x}_{1}, \frac{\mathbf{x}_{2}}{\beta}\right) e^{-\rho \mathbf{x}_{1}}, \quad \tilde{k}=\frac{k}{1-M^{2}}
\end{gathered}
$$

$$
\rho=-\frac{i k M}{1-M^{2}}, \quad \beta=\sqrt{1-M^{2}}
$$

Proof. The proof relies on simple but tedious computations which simply amounts to compute the derivatives of $\tilde{u}$ up to order 2 and arrange the results to obtain an Helmholtz equation. These computations are reported in Appendix A.

Remark 15. In practice, it is more convenient to approximate directly problem (74) instead of its equivalent Helmholtz problem (in $\tilde{u}$ ) since in this last one spurious oscillations might occur.

Proposition 6. Problem (74) admits a unique solution $u \in H^{1}(\Omega)$ for all $k \geq 1$. In addition, the stability property from Definition 2 is satisfied with $\alpha=0$.

Proof. Let us define $\tilde{u} \in H^{1}(\tilde{\Omega})$ as in Lemma 5. Since $\tilde{u}$ is defined as the solution to a standard Helmholtz problem, it is uniquely defined (see Hetmaniuk (2007) for instance). In addition, $\tilde{u}$ satisfies

$$
k\|\tilde{u}\|_{0, \tilde{\Omega}}+|\tilde{u}|_{1, \tilde{\Omega}}+k^{-1}|\tilde{u}|_{2, \tilde{\Omega}} \lesssim\|\tilde{f}\|_{0, \tilde{\Omega}}
$$

Then, one easily checks that

$$
\|u\|_{0, \Omega} \lesssim\|\tilde{u}\|_{0, \tilde{\Omega}}, \quad\|\tilde{f}\|_{0, \tilde{\Omega}} \lesssim\|f\|_{0, \Omega} \lesssim\|\tilde{f}\|_{0, \tilde{\Omega}}
$$

so that

Furthermore, we remark that

$$
k\|u\|_{0, \Omega} \lesssim\|f\|_{0, \Omega}
$$

$$
|u|_{1, \Omega} \lesssim|\tilde{u}|_{1, \Omega}+k\|\tilde{u}\|_{0, \Omega},
$$

hence using (75), we obtain

$$
|u|_{1, \Omega} \lesssim\|f\|_{0, \Omega}
$$


Similarly, for the estimate of the $H^{2}(\Omega)$ semi-norm, we notice that

$$
|u|_{2, \Omega} \lesssim|\tilde{u}|_{2, \Omega}+k|\tilde{u}|_{1, \Omega}+k^{2}\|\tilde{u}\|_{0, \Omega}
$$

and we conclude that

$$
|u|_{2, \Omega} \lesssim k\|f\|_{0, \Omega}
$$

using again (75).

5.1. Finite element discretization. The sesquilinear form associated with the convected Helmholtz equation (74) reads:

$$
\begin{aligned}
b_{k}(u, v) & =-k^{2}(u, v)+\left(1-M^{2}\right)\left(\frac{\partial u}{\partial \mathbf{x}_{1}}, \frac{\partial v}{\partial \mathbf{x}_{1}}\right)+\left(\frac{\partial u}{\partial \mathbf{x}_{2}}, \frac{\partial v}{\partial \mathbf{x}_{2}}\right) \\
& -2 i k M\left(\frac{\partial u}{\partial \mathbf{x}_{1}}, v\right)-\left\langle i k\left(1-M n_{1}\right) u, v\right\rangle_{\partial \Omega}
\end{aligned}
$$

for all $u, v \in H^{1}(\Omega)$.

In order to apply Theorem 2, we need to identify the adjoint operator. To do so, we consider $g \in L^{2}(\Omega)$ and assume that $v \in H^{1}(\Omega)$ satisfies $b_{k}(\phi, v)=(\phi, g)$ for all $\phi \in H^{1}(\Omega)$. We perform integration by parts on the left-hand side, and observe that

$$
\begin{cases}-k^{2} v-2 i k M \frac{\partial v}{\partial \mathbf{x}_{1}}-\left(1-M^{2}\right) \frac{\partial^{2} v}{\partial \mathbf{x}_{1}^{2}}-\frac{\partial^{2} v}{\partial \mathbf{x}_{2}}=g & \text { in } \Omega \\ \left(1-M^{2}\right) \frac{\partial v}{\partial \mathbf{x}_{1}} n_{1}+\frac{\partial v}{\partial \mathbf{x}_{1}} n_{2}+i k\left(1+M n_{1}\right) v= & \quad \text { on } \partial \Omega .\end{cases}
$$

Then, we take the complex conjugate to get

$$
\begin{cases}-k^{2} \bar{v}+2 i k M \frac{\partial \bar{v}}{\partial \mathbf{x}_{1}}-\left(1-M^{2}\right) \frac{\partial^{2} \bar{v}}{\partial \mathbf{x}_{1}^{2}}-\frac{\partial^{2} \bar{v}}{\partial \mathbf{x}_{2}^{2}}=\bar{g} & \text { in } \Omega \\ \left(1-M^{2}\right) \frac{\partial \bar{v}}{\partial \mathbf{x}_{1}} n_{1}+\frac{\partial \bar{v}}{\partial \mathbf{x}_{1}} n_{2}-i k\left(1+M n_{1}\right) \bar{v}= & 0 \quad \text { on } \partial \Omega .\end{cases}
$$

Hence, $\bar{v}$ is solution to the direct problem with an opposite Mach number and $\bar{g}$ as a righthand-side. We thus see that both $\mathcal{P}_{k}$ and $\mathcal{P}_{k}^{\star}$ satisfy the assumptions from Definitions 1 and 2 and therefore, Theorem 2 applies.

5.2. Numerical experiments. We consider problem (74) with a Mach number $M=0.4$ and $f=\mathbf{1}_{(1 / 2-1 / 16,1 / 2+1 / 16)^{2}}$. The problem is solved for various wavenumbers $k$.

We proceed as in Section 4.4 to assess the results of our theoretical analysis. As in this example the analytical solution to the problem is unknown, an "overkilling" finite element solution, computed on a very fine mesh, is used as reference instead. This accurate solution is also used to compute the best approximation $w_{h p}$.

The value $h^{\star}(k)$ designates the beginning of the asymptotic range for a given $k$, and is defined as in Section 4.4. Figure 5 shows the behaviour of $h^{\star}(k)$ with respect to $k$ for $\mathbb{P}_{1}$ and $\mathbb{P}_{2}$ elements. In both case, we observe that $h^{\star}(k) \sim k^{-1-1 / p}$, which is accordance with the condition provided in Theorem 2 and confirm that this condition is sharp.

\section{Application to the acoustic Helmholtz equation in heterogeneous media}

Here, we consider a polygonal domain $\Omega=(-2,2)^{2} \subset \mathbb{R}^{2}$, divided into three subdomains $\Omega_{q}, q=$ $1,2,3$, that represent distinct acoustic materials. Figure 6 depicts the considered geometrical configuration. The compressibility and density of each material are respectively denoted by $\beta_{q}$ and $\rho_{q}$ and take here the values

$$
\begin{array}{lll}
\beta_{1}=1, & \beta_{2}=10, & \beta_{3}=1000 \\
\rho_{1}=1, & \rho_{2}=0.5, & \rho_{3}=0.1 .
\end{array}
$$



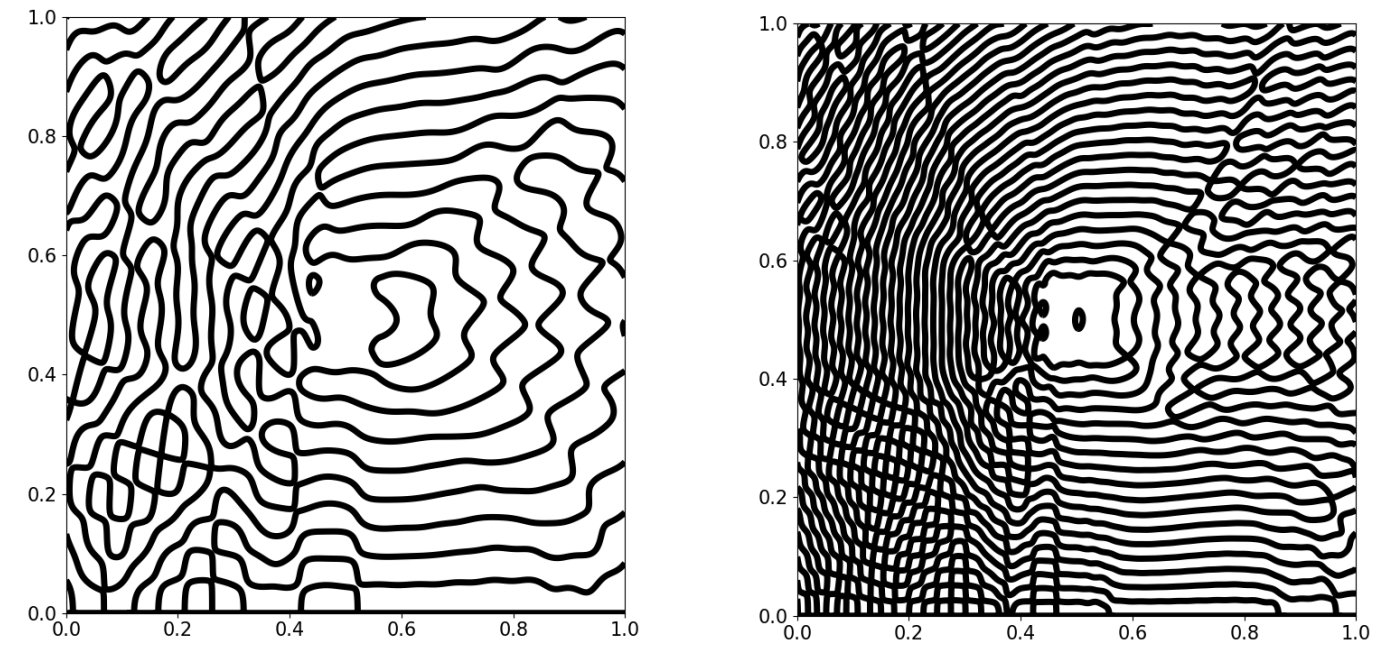

Figure 4. Zero-levelset curves of the real parts of solutions to the convected Helmholtz equation for $k=10$ (Left) and 20 (Right)
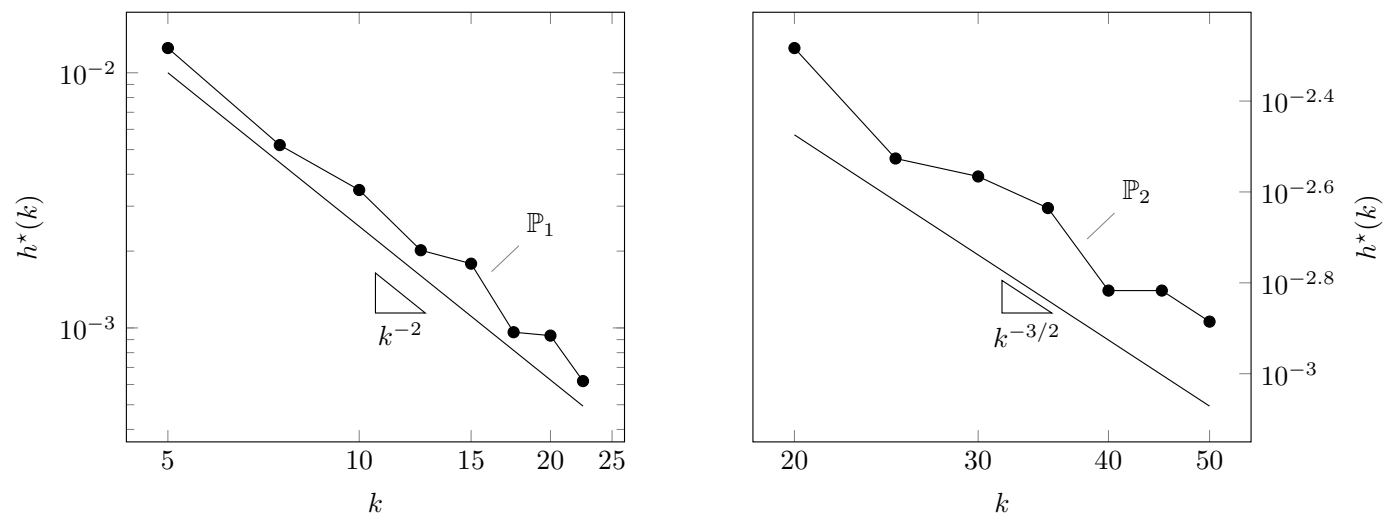

Figure 5. Convected Helmholtz equation: asymptotic ranges $h^{\star}(k)$ for $\mathbb{P}_{1}$ (left) and $\mathbb{P}_{2}$ (right) elements

Then, we consider the problem to find $u: \Omega \rightarrow \mathbb{C}$ such that

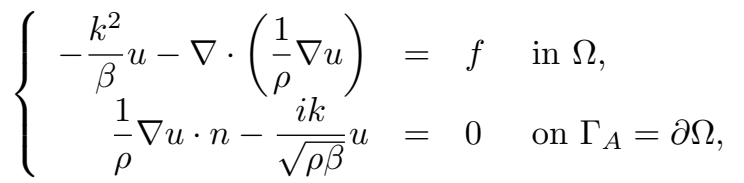

where $\left.\beta\right|_{\Omega_{q}}=\beta_{q},\left.\rho\right|_{\Omega_{q}}=\rho_{q}$, and $f \in L^{2}(\Omega)$ is a Gaussian load term centered at $\mathbf{y}=(0.0,-0.5)$. Specifically, we chose

$$
f(\mathbf{x})=\exp \left(-\frac{|\mathbf{x}-\mathbf{y}|^{2}}{\sigma^{2}}\right), \quad \text { with } \sigma=10^{-2} .
$$

Figure 7 represents the solution to (76) for two different frequencies.

Problem (76) enters into our framework with $\mathcal{L}_{0} u=\beta^{-1} \mathrm{u}, \mathcal{L}_{1} u=0, \mathcal{L}_{2} u=-\nabla \cdot\left(\rho^{-1} \nabla u\right)$, $\mathcal{B}_{A} u=\rho^{-1} \nabla u \cdot n$ and $A=(\rho \beta)^{-1 / 2}$. Since each subdomain has corners, problem (5) (with the previous choice of $\mathcal{L}_{2}$ and $\mathcal{B}_{A}$ ) may have singularities. They are described in Nicaise \& Sändig (1994a) for instance. In our configuration they appear at all interior corners. Whatever their 

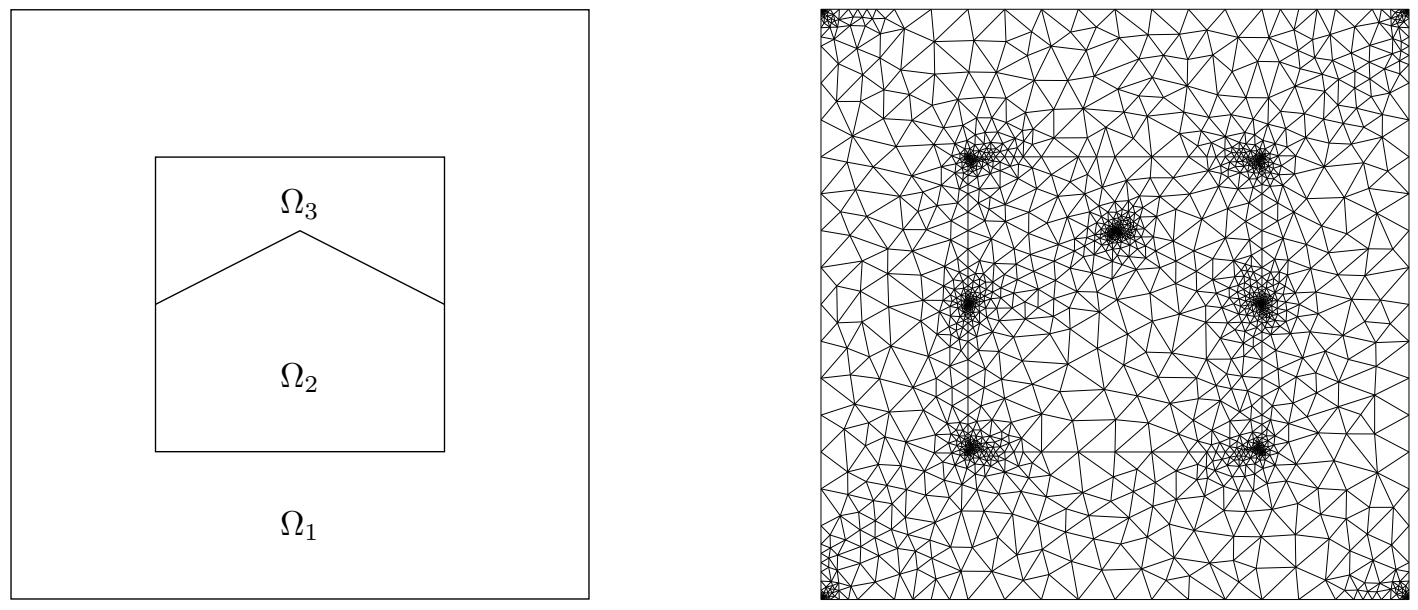

Figure 6. Left: Configuration of the acoustic Helmholtz problem. Right: Example of a refined mesh.
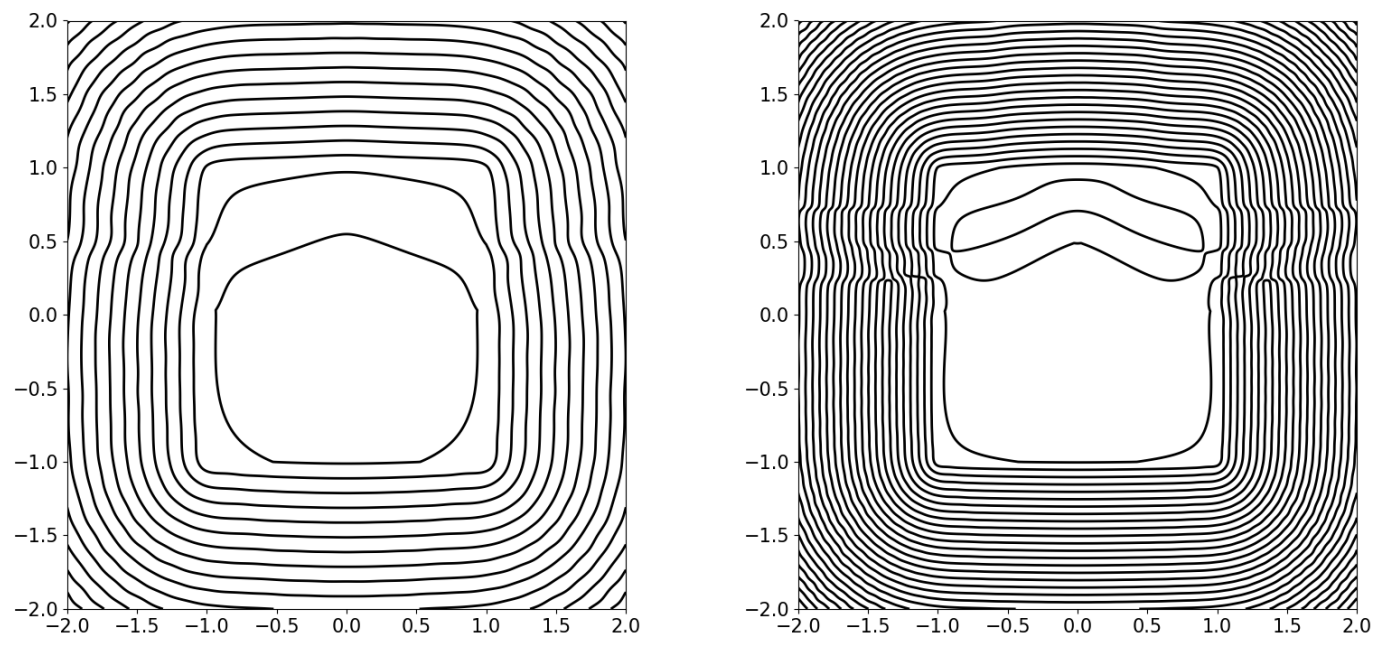

FiguRE 7. Zero-levelset curves of the real parts of solutions to the heterogeneous Helmholtz equation for $k=10 \pi$ (Left) and $20 \pi$ (Right)

expression, by Theorem 7.4 of Nicaise \& Sändig (1994b), the shift property from Definition 1 hold. Exactly as before, the construction of the singular spaces $\mathcal{T}_{c, j}^{\lambda}$ directly follows from a logarithmopolynomial resolution, see (Nicaise \& Sändig, 1994b, Lemma 7.1) or (Nicaise, 1993, §4.3, 4.4). On the other hand, it is established in Graham et al. (2018) that Problem (76) satisfies the $k$-stability property of Definition 2 with $\alpha=0$. Indeed, Problem (76) is a particular case of Definition 2.2 of Graham et al. (2018) and the coefficients $\beta$ and $\rho$ satisfies Condition 5.6 of Graham et al. (2018). As a result, Theorems 1 and 2 hold.

We employ the software triangle (Shewchuk, 1996) to generate the meshes. Specifically, for a given mesh size $h$, we impose the maximal area condition $|K| \leq h^{2} / 2$ by using the "-a" flag of triangle. In addition, we add artificial points near each corner that might generate a singularity in the input file given to triangle. More precisely, such points are placed at a distance $h / 1000$ of each corner. We also use the flag "-q33" of triangle to impose a minimal angle condition of 
$33^{\circ}$. Thus, we make sure that the mesh is properly graded close to each singular point. Figure 6 presents an example of such a mesh for $h=0.1$.

We follow the methodology previously introduced to compute $h^{\star}(k)$, using the "natural" norm

$$
\|\| v\left\|\left.\right|^{2}=k^{2}\right\| \beta^{-1 / 2} v\left\|_{0, \Omega}^{2}+\right\| \rho^{-1 / 2} \nabla v \|_{0, \Omega}^{2}, \quad v \in H^{1}(\Omega) .
$$

As shown in Figure 8, we numerically observe that $h^{\star}(k) \simeq k^{-1-1 / p}$ for $\mathbb{P}_{1}$ and $\mathbb{P}_{2}$ finite elements. It is in agreement with the developed theory, and shows that the main results are sharp.
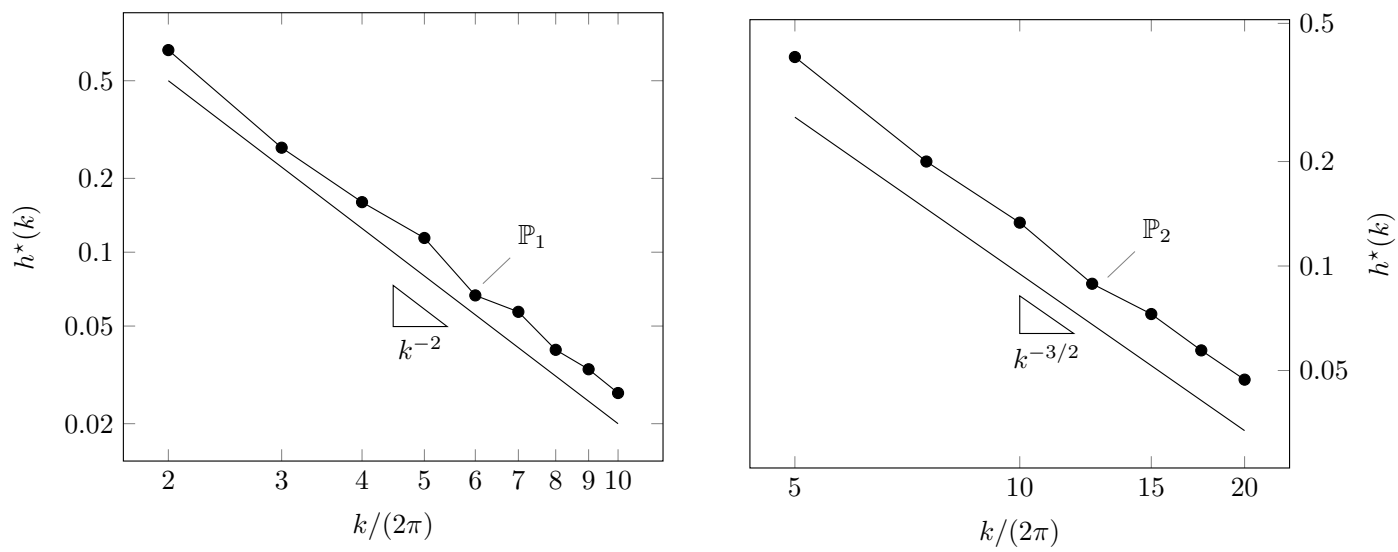

Figure 8. Heterogeneous Helmholtz equation: asymptotic ranges $h^{\star}(k)$ for $\mathbb{P}_{1}$ (left) and $\mathbb{P}_{2}$ (right) elements

\section{CONCLUSION}

We have presented a general approach to split up the solution of a wave propagation problem into a regular oscillating part and a $A^{2}$ (i.e. $H^{2}$ plus singularities close to corners) component whose norm can be controlled explicitly with respect to the wavenumber. For the case of the acoustic Helmholtz equation, this type of splitting is available in the literature (Esterhazy \& Melenk, 2012; Melenk \& Sauter, 2010, 2011). However, our approach to build the splitting is different. The main advantage of our construction is that it is valid for a large class of wave propagation operators, including problems with space-dependent wavespeed, and non-analytic domains. We highlight this by considering the elastodynamic system, the convected Helmholtz equation and the heterogeneous Helmholtz equation with piecewise constant coefficients.

We have taken advantage of the splitting to derive asymptotic error estimates for finite element discretizations. In this analysis, we consider finite element spaces of arbitray high, but fixed, discretization order $p$. The main conclusion is that the finite element solution is quasi-optimal under the condition that $k h$ and $k^{p+1} h^{p}$ are small enough, where $k$ and $h$ are the wavenumber and the mesh size. Such a result was already established in Esterhazy \& Melenk (2012), Melenk \& Sauter (2010) and Melenk \& Sauter (2011) for the case of the acoustic Helmholtz equation in homogeneous media, so that our result is a generalization to more complex wave propagation problems.

We performed numerical experiments for the case of the elastodynamic system, the convected Helmholtz equation and the heterogeneous Helmholtz equation. These examples indicate that our theoretical analysis is sharp.

The analysis presented in this paper focuses on finite element discretization of fixed discretizations order $p$. In future works, we would like to explicitly track the $p$ dependence in the constants to consider fully $h p$ finite element methods. On the other hand, another extension would be to consider general three dimensional domains and partition that contains sharp edges. 


\section{Appendix A. Proof of Lemma 5}

Let $v\left(\mathbf{x}_{1}, \mathbf{x}_{2}\right)=u e^{-\rho \mathbf{x}_{1}}$, so that $u\left(\mathbf{x}_{1}, \mathbf{x}_{2}\right)=v\left(\mathbf{x}_{1}, \mathbf{x}_{2}\right) e^{\rho \mathbf{x}_{1}}$. By elementary calculations, we show that

$$
\begin{aligned}
-k^{2} u-2 i k M \frac{\partial u}{\partial \mathbf{x}_{1}}-\left(1-M^{2}\right) \frac{\partial^{2} u}{\partial \mathbf{x}_{1}^{2}}-\frac{\partial^{2} u}{\partial \mathbf{x}_{2}^{2}}= & -\left(k^{2}+2 i k M \rho+\left(1-M^{2}\right) \rho^{2}\right) v e^{\rho \mathbf{x}_{1}} \\
& -\left(2 i k M+2(1-M)^{2} \rho\right) \frac{\partial v}{\partial \mathbf{x}_{1}} e^{\rho \mathbf{x}_{1}} \\
& -\left(1-M^{2}\right) \frac{\partial^{2} v}{\partial \mathbf{x}_{1}^{2}} e^{\rho \mathbf{x}_{1}}-\frac{\partial^{2} v}{\partial \mathbf{x}_{2}^{2}} e^{\rho \mathbf{x}_{1}}
\end{aligned}
$$

so that

$$
-\left(k^{2}+2 i k M \rho+\left(1-M^{2}\right) \rho^{2}\right) v-2\left(i k M+\left(1-M^{2}\right) \rho\right) \frac{\partial v}{\partial \mathbf{x}_{1}}-\left(1-M^{2}\right) \frac{\partial^{2} v}{\partial \mathbf{x}_{1}^{2}}-\frac{\partial^{2} v}{\partial \mathbf{x}_{2}^{2}}=f e^{-\rho \mathbf{x}_{1}} .
$$

We now select the value of $\rho$ to guarantee that

$$
i k M+\left(1-M^{2}\right) \rho=0,
$$

hence we take $\rho=-i k M /\left(1-M^{2}\right)$. With this choice, we get

$$
\begin{aligned}
k^{2}+2 i k M \rho+\left(1-M^{2}\right) \rho^{2} & =k^{2}-2 \frac{(i k M)^{2}}{1-M^{2}}+\frac{(-i k M)^{2}}{1-M^{2}} \\
& =k^{2}+\frac{M^{2} k^{2}}{1-M^{2}} \\
& =\frac{k^{2}}{1-M^{2}} .
\end{aligned}
$$

As a result, $v$ satisfies

$$
-\frac{k^{2}}{1-M^{2}} v-\left(1-M^{2}\right) \frac{\partial^{2} v}{\partial \mathbf{x}_{1}^{2}}-\frac{\partial^{2} v}{\partial \mathbf{x}_{2}^{2}}=f e^{-\rho \mathbf{x}_{1}} .
$$

On the boundary, it holds that

$$
\begin{aligned}
0 & =\left(1-M^{2}\right) \frac{\partial u}{\partial \mathbf{x}_{1}} n_{1}+\frac{\partial u}{\partial \mathbf{x}_{2}} n_{2}-i k\left(1-M n_{1}\right) u \\
& =\left(1-M^{2}\right) \frac{\partial v}{\partial \mathbf{x}_{1}} e^{\rho \mathbf{x}_{1}} n_{1}+\frac{\partial v}{\partial \mathbf{x}_{2}} e^{\rho \mathbf{x}_{1}} n_{2}+\left(\left(1-M^{2}\right) \rho-i k\left(1-M n_{1}\right)\right) v e^{\rho \mathbf{x}_{1}}
\end{aligned}
$$

Hence

$$
\left(1-M^{2}\right) \frac{\partial v}{\partial \mathbf{x}_{1}} n_{1}+\frac{\partial v}{\partial \mathbf{x}_{2}} n_{2}+\left(\left(1-M^{2}\right) n_{1} \rho-i k\left(1-M n_{1}\right)\right) v=0 .
$$

As simple calculations yield

$$
\left(1-M^{2}\right) \rho-i k\left(1-M n_{1}\right)=-i k M n_{1}-i k+i k M n_{1}=-i k,
$$

the previous boundary condition is equivalent to

$$
\left(1-M^{2}\right) \frac{\partial v}{\partial \mathbf{x}_{1}} n_{1}+\frac{\partial v}{\partial \mathbf{x}_{2}} n_{2}-i k v=0 .
$$

Now, we set $v\left(\mathbf{x}_{1}, \mathbf{x}_{2}\right)=\tilde{u}\left(\mathbf{x}_{1}, \beta \mathbf{x}_{2}\right)$, so that

$$
\frac{\partial^{2} v}{\partial \mathbf{x}_{2}^{2}}=\beta^{2} \frac{\partial^{2} \tilde{u}}{\partial \tilde{\mathbf{x}}_{2}^{2}}\left(\mathbf{x}_{1}, \beta \mathbf{x}_{2}\right)
$$

and

$$
\frac{\partial v}{\partial \mathbf{x}_{2}} n_{2}=\beta^{2} \frac{\partial \tilde{u}}{\partial \tilde{\mathbf{x}}_{2}}\left(\mathbf{x}_{1}, \beta \mathbf{x}_{2}\right) \tilde{n}_{2} .
$$

Setting $\beta=\sqrt{1-M^{2}}$ and dividing both equations by $1-M^{2}$, we obtain that

$$
-\left(\frac{k}{1-M^{2}}\right)^{2} \tilde{u}-\tilde{\Delta} \tilde{u}=\frac{1}{1-M^{2}} f e^{-\rho \mathbf{x}_{1}}
$$


in $\tilde{\Omega}$ and

$$
\tilde{\nabla} \tilde{u} \cdot \tilde{n}-\frac{k}{1-M^{2}} \tilde{u}=0
$$

on $\partial \tilde{\Omega}$.

\section{ACKNOWLEDGEMENTS}

T. Chaumont-Frelet has received funding from the Project of the Spanish Ministry of Economy and Competitiveness with reference MTM2016-76329-R, the BCAM "Severo Ochoa" accreditation of excellence SEV-2013-0323, and the Basque Government through the BERC 2014-2017 program.

\section{REFERENCES}

1. Alabau, F. \& Komornik, V. (1998) Boundary observability, controllability, and stabilization of linear elastodynamic systems. SIAM J. Control. Optim., 37, 521-542.

2. BABUŠKA, I. (1970) The finite element method for elliptic equations with discontinuous coefficients. Computing, 5, 207-213.

3. BarucQ, H., Boillot, L., Calandra, H. \& Diaz, J. (2014) Absorbing boundary condition for 2d tilted transverse isotropic elastic media. ESAIM: Proceedings and Surveys, 45, 400-409.

4. Bécache, E., Dhia, A. B.-B. \& Legendre, G. (2004) Perfectly matched layers for the convected Helmholtz equation. SIAM J. Numer. Anal., 42, 409-433.

5. Bernardi, C. (1989) Optimal finite-element interpolation on curved domains. SIAM J. Numer. Anal., 26, 1212-1240.

6. Boillot, L., Barucq, H., Diaz, J. \& Calandra, H. (2015) Absorbing boundary conditions for 3d elastic TTI modeling. SEG New Orleans Meeting, 535-540.

7. Brown, D. \& Gallistl, D. (2016) Multiscale sub-grid correction method for time-harmonic high-frequency elastodynamics with wavenumber explicit bounds. arXiv:1608.04243.

8. Chavent, G., Papanicolaou, G., Sacks, P. \& Symes, W. (2012) Inverse Problems in Wave Propagation. Springer.

9. Ciarlet, P. G. (2010) On Korn's inequality. Chin. Ann. Math. Ser. B, 31, 607-618.

10. Clayton, R. \& Engquist, B. (1977) Absorbing boundary conditions for acoustic and elastic wave equations. Bulletin of the Seismological Society of America, 67, 1529-1540.

11. Colton, D. \& Kress, R. (2012) Inverse Acoustic and Electromagnetic Scattering Theory. Springer.

12. Costabel, M., Dauge, M. \& Nicaise, S. (http://hal.archives-ouvertes.fr/hal-00453934/en/ Online version of Chapters 1 to 5. (2010)) Corner Singularities and Analytic Regularity for Linear Elliptic Systems. Part I: Smooth domains. Corner Singularities and Analytic Regularity for Linear Elliptic Systems. Part I: Smooth domains.

13. Cummings, P. \& Feng, X. (2006) Sharp regularity coefficient estiamtes for complex-valued acoustic and elastic Helmholtz equations. Math. Mod. Meth. Appl. Sci., 16, 139-160.

14. Darbas, M., Geuzaine, C. \& Mattesi, V. (2017) High-order absorbing boundary conditions for $2 \mathrm{~d}$ elastic scattering problems. in preparation. Univ. Liège.

15. Dauge, M. (1988) Elliptic boundary value problems on corner domains - smoothness and asymptotics of solutions. Lecture Notes in Mathematics, vol. 1341. Berlin: Springer.

16. Dauge, M., Nicaise, S., Bourlard, M. \& Lubuma, J. M.-S. (1990) Coefficients des singularités pour des problèmes aux limites elliptiques sur un domaine à points coniques. I. Résultats généraux pour le problème de Dirichlet. RAIRO Modél. Math. Anal. Numér., 24, 27-52.

17. Duvaut, G. \& Lions, J.-L. (1972) Les inéquations en mécanique et en physique. Dunod, Paris, pp. $x x+387$. Travaux et Recherches Mathématiques, No. 21.

18. Esterhazy, S. \& Melenk, J. (2012) On stability of discretizations of the Helmholtz equation. Lecture Notes in Computational Science and Engineering. Springer-Verlag Berlin Heidelberg, pp. 285-324.

19. Graham, I., Pembery, O. \& Spence, E. (2018) The Helmholtz equation in heterogeneous media: a priori bounds, well-posedness, and resonances. arXiv preprint arXiv:1801.08095. 
20. Grisvard, P. (1985) Elliptic problems in nonsmooth domains. Monographs and Studies in Mathematics, vol. 24. Boston-London-Melbourne: Pitman.

21. Grisvard, P. (1986) Problèmes aux limites dans les polygones. Mode d'emploi. (Boundary value problems in plane polygons. Instructions for use). Bull. Dir. Etud. Rech., Sér. C, 1, 21-59.

22. Hetmaniuk, U. (2007) Stability estimates for a class of Helmholtz problems. Commun. Math. Sci., 5, 665-678.

23. Huang, F. L. (1985) Characteristic conditions for exponential stability of linear dynamical systems in Hilbert spaces. Ann. Differential Equations, 1, 43-56.

24. Ihlenburg, F. \& BABUŠKA, I. (1995) Finite element solution of the Helmholtz equation with high wave number part i: the h-version of the fem. Computers Math. Applic., 30, 9-37.

25. Ihlenburg, F. \& BABUŠKA, I. (1997) Finite element solution of the Helmholtz equation with high wave number part ii: the h-p version of the fem. SIAM J. Numer. Anal., 34, 315-358.

26. Kondrat'Ev, V. A. (1967) Boundary value problems for elliptic equations on domains with conical or angular points. Trudy Moskov. Mat. Obshch., 16, 209-292. In Russian.

27. Kozlov, V. A., Maz'ya, V. G. \& Rossmann, J. (2001) Spectral Problems Associated with Corner Singularities of Solutions to Elliptic Equations. Mathematical Surveys and Monographs, 85. Providence, RI: American Mathematical Society.

28. Lagnese, J. (1983) Boundary stabilization of linear elastodynamic systems. SIAM J. Control Optim., 21, 968-984.

29. Lions, J.-L. \& Magenes, E. (1968) Problèmes aux limites non homogènes et applications. Vol. 1. Travaux et Recherches Mathématiques, No. 17. Paris: Dunod, pp. xx +372 .

30. Lubuma, J. M.-S. \& NicAise, S. (1995) Dirichlet problems in polyhedral domains II: approximation by FEM and BEM. J. Comp. Appl. Math., 61, 13-27.

31. Lysmer, J. \& Kuhlemeyer, R. (1969) Finite dynamic model for infinite media. J. Eng. Mech. Div., ASCE, 95, 859877.

32. MaZ'ya, V. \& Rossmann, J. (2010) Elliptic equations in polyhedral domains. Mathematical Surveys and Monographs, vol. 162. American Mathematical Society, Providence, RI, pp. viii+608.

33. Melenk, J. M. \& Sauter, S. (2018) Wavenumber-explicit hp-FEM analysis for Maxwell's equations with transparent boundary conditions. arXiv preprint arXiv:1803.01619v1.

34. Melenk, J. \& Sauter, S. (2010) Convergence analysis for finite element discretizations of the Helmholtz equation with Dirichlet-to-Neumann boundary conditions. Mathematics of Computation, 79, 1871-1914.

35. Melenk, J. \& Sauter, S. (2011) Wavenumber explicit convergence analysis for Galerkin discretizations of the Helmholtz equation. SIAM J. Numer. Anal., 49, 1210-1243.

36. NeČAs, J. (1967) Les méthodes directes en théorie des équations elliptiques. Prague: Academia.

37. Nicaise, S. (1993) Polygonal Interface Problems. Methoden und Verfahren der mathematischen Physik, vol. 39. Frankfurt/M.: Peter Lang GmbH, Europäischer Verlag der Wissenschaften.

38. Nicaise, S. (2003) Stability and controllability of an abstract evolution equation of hyperbolic type and concrete applications. Rendiconti di Matematica Serie VII, 23, 83-116.

39. Nicaise, S. \& SÄndig, A.-M. (1994a) General interface problems I. Math. Methods in the Appl. Sc., 17, 395-429.

40. Nicaise, S. \& SÄNdig, A.-M. (1994b) General interface problems II. Math. Methods in the Appl. Sc., 17, 431-450.

41. Prüss, J. (1984) On the spectrum of $C_{0}$-semigroups. Trans. Amer. Math. Soc., 284, 847-857.

42. Schatz, A. (1974) An observation concerning Ritz-Galerkin methods with indefinite bilinear forms. Mathematics of Computation, 28, 959-962.

43. Shewchuk, J. (1996) Triangle: engineering a 2D quality mesh generator and Delaunay triangulator. Applied computational geometry towards geometric engineering. Springer, pp. 203-222. 